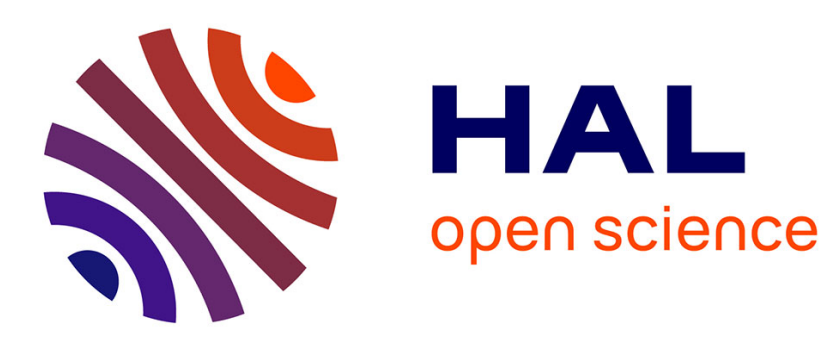

\title{
Eléments pour l'ébauche d'une socio-histoire du groupe professionnel infirmier. Un fil conducteur: la formation des infirmières et de leurs cheffes
}

Sophie Divay, Lucile Girard

\section{- To cite this version:}

Sophie Divay, Lucile Girard. Eléments pour l'ébauche d'une socio-histoire du groupe professionnel infirmier. Un fil conducteur: la formation des infirmières et de leurs cheffes. Recherche en soins infirmiers, 2019. hal-02986475

\section{HAL Id: hal-02986475 \\ https://hal.science/hal-02986475}

Submitted on 3 Nov 2020

HAL is a multi-disciplinary open access archive for the deposit and dissemination of scientific research documents, whether they are published or not. The documents may come from teaching and research institutions in France or abroad, or from public or private research centers.
L'archive ouverte pluridisciplinaire HAL, est destinée au dépôt et à la diffusion de documents scientifiques de niveau recherche, publiés ou non, émanant des établissements d'enseignement et de recherche français ou étrangers, des laboratoires publics ou privés. 


\title{
Sophie Divay, Lucile Girard
}

Eléments pour l'ébauche d'une socio-histoire du groupe professionnel infirmier.

Un fil conducteur : la formation des infirmières et de leurs cheffes

Recherche en Soins Infirmiers, déc, $\mathrm{n}^{\circ} 139, \mathbf{2 0 1 9}, \mathrm{pp} .64-83$

\section{Titre :}

Eléments pour l'ébauche d'une sociohistoire du groupe professionnel infirmier. Un fil conducteur : la formation des infirmières et de leurs cheffes

\section{Title:}

Draft elements to the socio-history of the nurses' professional group. Main thread: the nurses and senior nurses' training.

\section{Résumé}

Cet article retrace certaines des dynamiques du groupe professionnel infirmier depuis son émergence à la fin du $\mathrm{XIX}^{\mathrm{e}}$ siècle en France. Notre propos suit le fil conducteur des évolutions des dispositifs de formation mis en place pour les infirmières, puis pour leurs cheffes. Les éléments rassemblés ici œuvrent pour la constitution d'une socio-histoire du monde infirmier et de la complexité de ses dynamiques. Elles se manifestent dans un premier temps à travers les luttes entre les promoteur.e.s de définitions divergentes, voire antagonistes, de l' «infirmière moderne », chacun·e proposant des modèles de fabrication (formation et socialisation de ces soignantes) plus ou moins contrastés. L'entrée en scène progressive du segment des cheffes d'infirmières contribue à l'avènement d'une des premières formes d'autonomisation et d'affirmation du groupe professionnel qui au fil du temps va stratégiquement adhérer, en les combinant, à différentes idéologies (des principes humanistes des soins infirmiers aux orientations managériales gestionnaires, en passant par une conception biomédicalisée du travail infirmier). Les analyses présentées ici s'appuient sur un travail de thèse soutenue en 2019 et sur de nombreuses enquêtes menées depuis des années tant dans les instituts de formation des infirmières que sur leurs lieux de travail à l'hôpital.

\section{Mots clés}

Socio-histoire ; groupes professionnels ; infirmières ; formation ; hôpital

\begin{abstract}
This article retraces some of the dynamics of the nurses' professional group since its advent at the end of the XIX ${ }^{\text {th }}$ century in France. The guiding thread we will follow is the evolution of the institutional education of nurses and senior nurses. Through a socio-historical approach, we will understand this intricate process. It takes the form of struggles between different and even opposite views of "the modern nurse". Each view puts forward its own education and socialization model. The constitution of a new segment - the senior nurses -participates in the group's self-governance. The professional group will strategically share many kinds of ideology (like the humanist aspects of care, the biomedical vision of a nurse's work, or managerial guidelines). We based our work on a PhD thesis presented in 2018 and on various surveys conducted both among training institutions and hospital work places.
\end{abstract}




\section{Keywords}

Socio-history; professional groups; nurses; training; hospital

\section{1- Introduction}

En France, l'émergence de la « profession » d'infirmière ${ }^{1}$ se produit à partir du début du $\mathrm{XX}^{\mathrm{e}}$ siècle; son histoire peut être retracée à travers un examen chronologique des textes officiels ayant progressivement contribué à consolider sa structuration au sein d'un environnement où elle a dû faire sa place, notamment aux côtés des médecins, et entre autres au sein des hôpitaux. En 1902, une circulaire ${ }^{2}$, signée par Emile Combes, alors président du Conseil et ministre de l'Intérieur et des Cultes, rappelle aux préfets l'« obligation absolue » (dont le nonrespect $n$ 'est toutefois pas sanctionné) à laquelle sont tenues les commissions administratives de chaque hôpital de recruter un « personnel capable, soit en créant elle[s]-même[s] une école d'infirmière, soit en traitant avec un autre hôpital pourvu d'une telle école ». Il faut encore attendre vingt ans pour qu'un décret ${ }^{3}$ promulgue la création des «brevets de capacité professionnelle conférant le titre d'infirmière diplômée de l'Etat français », mais sans rendre pour autant obligatoire l'embauche d'infirmières diplômées. Pendant de longues années encore nombre d'infirmières officieront sans avoir suivi de formation dans une école et sans être diplômées. Le titre ne sera légalement protégé qu'à partir de $1943^{4}$. Deux bonds dans le temps, nous propulsent tout d'abord en $2009^{5}$, année de la parution du nouveau référentiel de formation infirmière octroyant non seulement un diplôme d'Etat d'infirmière mais aussi le grade de licence, diplôme de niveau II (bac +3), puis au mois d'août 2019 au cours duquel le master d'infirmières en pratiques avancées (IPA) ${ }^{6}$ est mis en place, ouvrant ainsi la voie à l'instauration d'un cursus universitaire complet, jusqu'au doctorat en soins infirmiers.

Bien que très lacunaire, cette énumération d'étapes démontre qu'en l'occurrence le processus de «professionnalisation» des infirmières repose en grande partie sur la création et le développement d'une formation institutionnellement organisée et réglementée. Encore faut-il s'entendre sur le sens des mots. Ce constat concorde en fait avec les perspectives fonctionnalistes de la sociologie des professions selon lesquelles la «professionnalisation », c'est-à-dire le passage pour une activité du simple statut d'occupation à celui plus noble de profession, se décline en plusieurs phases ordonnées. Citant Wilensky (2), un des théoriciens de ce courant sociologique, Dubar (3) dresse la liste des caractéristiques qu'une profession doit successivement revêtir :

«1- être exercée à plein temps ;

\footnotetext{
${ }^{1}$ Au regard de la proportion importante de femmes dans la profession - toujours plus de $86 \%$ au $1^{\text {er }}$ janvier 2018 d'après la Directions de la recherche, des études, de l'évaluation et des statistiques (DREES) - nous faisons le choix de féminiser le groupe professionnel.

2 Circulaire Combes, parue au Journal Officiel de République française le 30 octobre 1902, adressée aux préfets au sujet de l'application de la loi du 15 juillet 1893 sur l'assistance médicale gratuite et la création d'écoles d'infirmière.

3 Décret du 17 juin 1922, portant création du brevet de capacité professionnelle et conférant le titre d'infirmière diplômée de l'Etat français.

$4 C f$. Loi du 15 juillet 1943, créant l'obligation pour les établissements hospitaliers de recruter un personnel qualifié, avec des mesures propres à assurer la protection du titre d'infirmière. La loi nº46-330 du 8 avril 1946 relative à l'exercice des professions d'assistantes ou d'auxiliaires de service social et d'infirmières ou d'infirmiers, parue au JORF le 9 avril 1946, reprend les articles de 1943 dans le Code de la santé publique. Le diplôme est obligatoire, mais prévoit des mesures dérogatoires (1).

5 Arrêté du 31 juillet 2009 relatif au diplôme d'Etat d'infirmier

6 Décret $n^{\circ}$ 2019-835 du 12 août 2019 relatif à l'exercice infirmier en pratique avancée et à sa prise en charge par l'assurance maladie.
} 
2- comporter des règles d'activité ;

3- comprendre une formation et des écoles spécialisées ;

4- posséder des organisations professionnelles ;

5- comporter une protection légale du monopole ;

6- avoir établi un code de déontologie. » (3, p.90)

De nombreux auteurs (sociologues, mais aussi historiens) ont souscrit à ce modèle pour étudier les évolutions des (vraies) professions, la plus emblématique de toutes étant celle de médecin ${ }^{7}$. Toutefois, ce modèle a été critiqué, d'une part, pour son manque de distanciation vis-à-vis des discours produits par les «professions » elles-mêmes sur elles-mêmes, moins réalistes que propagandistes, et, d'autre part, parce qu'il ne concerne qu'un nombre limité de catégories de travailleurs, exerçant souvent en libéral, hautement qualifiés, jouissant d'un fort prestige social et d'une grande valeur économique. Cette distinction (dans tous les sens du terme) s'avère pratiquement trop rigide et conduit à établir des sous-catégorisations entre les professions et les occupations, certains auteurs parlant de «quasi-profession », «semiprofession ", voire même d' " occupation en voie de professionnalisation ». Afin d'échapper à la polysémie du terme de profession, «objet de constants débats [qui] ne possède pas de définition universellement admise » $(4$, p.161), des sociologues ont préféré recourir à l'expression «groupes professionnels» qui permet de contourner ces difficultés «en élargissant le champ d'analyse au-delà du périmètre étroit des professions libérales ou réglementées, mais sans en diluer les significations. Elle désigne alors des ensembles de travailleurs exerçant une activité ayant le même nom, et par conséquent dotés d'une visibilité sociale, bénéficiant d'une identification et d'une reconnaissance, occupant une place différenciée dans la division sociale du travail, et caractérisés par une légitimité symbolique. Ils ne bénéficient pas nécessairement d'une reconnaissance juridique, mais du moins d'une reconnaissance de fait, largement partagée et symbolisée par leur nom, qui les différencie des autres activités professionnelles. En l'absence de réglementation et de codification formelles, les groupes professionnels sont des ensembles flous soumis à des changements continus, caractérisés à la fois par des contours évolutifs et une hétérogénéité interne. » $(5$, p.20)

Dans cette perspective, les groupes professionnels ne sont plus appréhendés à l'aune du questionnement réducteur sur leur éventuelle «professionnalisation», visant à déterminer si oui ou non une activité parvient à atteindre définitivement le stade envié d'une profession, comme si le sens de l'histoire était fixé d'avance, ignorant les dynamiques professionnelles dont son cours est jonché, "c'est-à-dire les processus d'émergence, de différenciation et d'autonomie d'activités professionnelles, et, plus largement, des mouvements diversifiés, ambigus et contradictoires de transformation des activités professionnelles (émergence, identification, délimitation, catégorisation, légitimation, invalidation, érosion, segmentation, destruction et disparition...) » (5,p.20). Un groupe professionnel peut certes connaître une fin, notamment à travers sa mort (6), mais de son émergence (moment difficile à situer car jamais uniquement repérable par le biais d'une date), à son terme, son histoire est composée d'événements peu prévisibles, entre autres dus au croisement, à l'entremêlement, l'entrechoc d'initiatives internes et externes, plus ou moins volontaires et stratégiques.

Après cette clarification de l'angle d'approche théorique adopté dans cet article, se réclamant d'une sociologie des groupes professionnels, il convient encore de préciser le choix des données exploitées. Si notre propos s'est ouvert sur la convocation de quelques textes de loi, nous avons pris soin d'en mentionner l'insuffisance. Plus précisément, il faut souligner que si les textes peuvent être considérés comme des signaux, des indices, ils ne sauraient constituer des matériaux dont on peut se satisfaire. A titre d'exemple, Schweyer (7) souligne que

7 Profession avec laquelle les infirmières entretiennent des liens empiriques complexes et ambigus, mais cet aspect est un sujet en soi qui pourrait faire l'objet d'un article à part entière. 
l'histoire des cadres soignants est souvent construite à partir des textes officiels, mais que si ces textes «attestent d'une reconnaissance officielle des fonctions d'encadrement. Pour autant, ils renseignent peu sur la réalité des activités et encore moins sur la constitution d'un groupe professionnel et d'une identité de cadre. La publication d'un décret créant une fonction de cadre ne peut être considérée comme l'acte de naissance de cette fonction, souvent déjà mise en place (on dirait aujourd'hui expérimentée) dans des établissements novateurs. Elle marque cependant une reconnaissance officielle et le début d'un mouvement de généralisation à l'ensemble des établissements des fonctions de cadres. La création d'un diplôme et d'un statut permet-elle la constitution d'un nouveau groupe professionnel ? » (7, p.17). En l'occurrence, les textes (décrets, arrêtés, circulaires, article du code de la Santé, référentiels, etc.) seront pris comme des points de repère temporels mettant en lumière les évolutions des dispositifs de formation des infirmières, et ces dispositifs seront considérés comme des révélateurs des dynamiques du groupe professionnel infirmier, non pas afin de mesurer son «degré de professionnalisation» mais de mettre en évidence les logiques sociales, orientations théoriques, choix politiques, définitions identitaires conçus par certains membres du groupe professionnel (non homogène) ainsi que par d'autres acteurs influents extérieurs au groupe à différents moments charnières de son histoire.

La lecture des évolutions d'un groupe professionnel à travers ses appareils de formation (§.1), ses savoirs transmis, ses procédures de certification et de qualification est bien connue en sociologie car elle renseigne sur un des moyens mis en œuvre pour améliorer la structuration d'un groupe professionnel, sa reconnaissance entre autres par l'Etat et son positionnement visà-vis des autres groupes professionnels voisins et de ceux qui se sont créés par segmentation interne. Nous compléterons cette exploration classique avec une focale sur les points de vue et actions collectives ou individuelles de membres de «l'élite » du groupe professionnel (§.2), c'est-à-dire les «cheffes » (surveillantes, cadres de santé, formatrices) qui ont contribué notamment à travers leurs définitions de l'infirmière, et donc de ses instances de formation et socialisation, à la construction de leur milieu professionnel. Ce faisant, nous insisterons sur la prise en compte, souvent négligée en sociologie, de l'importance de la constitution d'une « chefferie » inscrite dans une architecture hiérarchique stratifiée, venant s'ajouter au rang des moyens et instruments qui participent à la construction et consolidation d'un groupe professionnel dans le cadre des actions et luttes qu'il développe au sein d'une écologie liée ${ }^{8}$ (8) pour y faire, y conserver, voire y étendre sa place. La constitution de cet espace ordonné et hiérarchisé se rencontre de façon plus marquée dans les organisations, en l'occurrence dans les hôpitaux, où la condition d'exercice salariée soumet les travailleurs à un ordre institutionnel et organisationnel en grande partie réglé par l'Etat, tout à la fois soumis et porteur depuis plusieurs décennies (9) de la logique du new public management, favorisant la mutation des cadres soignants en managers-gestionnaires ( $\$ 3)$.

Encadré méthodologique : matériel et méthode

Cet article prend appui sur une thèse portant sur la formation des infirmières (10). Essentiellement qualitative, l'enquête que nous mobiliserons ici est composée de huit entretiens réalisés avec des formatrices d'un IFSI de l'est de la France. Nous avons porté notre attention sur les principales tâches dont elles sont chargées, sur leurs rapports avec les étudiantes, ainsi que

8 Abbott donne cette définition du concept d' «écologie liée » (il utilise le terme de «profession », en tant qu'américain moins sensible aux différenciations faites en France) : «Les professions, en concurrence les unes avec les autres, aspirent à se développer, s'emparant de telle ou telle sphère de travail qu'elles transforment ensuite en "juridiction" au moyen de savoirs professionnels et de revendications destinées à obtenir une légitimité auprès des pouvoirs publics. Un tel système est étroitement conditionné par la concurrence. Tout ce qui survient au sein d'une profession a des répercussions sur les professions voisines et se traduit soit par des développements soit par des défaites. » (8,p.29) 
sur leurs représentations de leur activité. A ces entretiens, nous avons ajouté l'analyse de diverses matériaux documentaires afin de retracer la socio-histoire du groupe professionnel infirmier: des textes de lois, utilisés comme des marqueurs de transformations ayant débuté en amont; les programmes de formation, pour mettre en perspective la structuration des discours professionnels (11); des articles de revues professionnelles, pour comprendre l'argumentation développée par chacun des types de protagonistes, infirmières et médecins; et enfin des données de seconde main, présentes dans différentes recherches historiques menées sur les infirmières.

Ont également été mobilisés d'autres enquêtes menées depuis plusieurs années dans les instituts de formation des cadres de santé, auprès des formatrices et des étudiantes, ainsi qu'au sein d'établissements de santé publics de taille et de statut différents, allant de l'hôpital local en zone rurale au CHU de l'AP-HP. Une analyse de la littérature professionnelle a également réalisée, notamment sur les écrits des intellectuelles infirmières et sur des séries de revues professionnelles destinées aux cadres de santé (12-15).

\section{2- La construction du groupe professionnel infirmier par l'institutionnalisation d'une formation initiale très lentement placée sous le contrôle de l'État}

A la fin du $\mathrm{XIX}^{\mathrm{e}}$ siècle, deux éléments principaux vont se conjuguer pour donner naissance à la catégorie que nous connaissons aujourd'hui sous le nom d'infirmière : les progrès de la médecine, et notamment la découverte des microbes et de la notion de contagion inhérente à la «révolution pasteurienne» d'une part; et d'autre part, la tendance anticléricale du gouvernement de la III $^{\mathrm{e}}$ République qui aboutira à la proclamation de la loi de séparation de l'Église et de l'État en 1905 et, pour ce qui nous intéresse, à la disparition très progressive des religieuses dans les établissements de soins. Nous verrons, toutefois, que la combinaison de ces éléments n'a pas mécaniquement entraîné l'émergence d'un nouveau groupe professionnel. Cette dernière résulte, au contraire, de processus historiques complexes, fruits de luttes entre des visions différentes, voire opposées, de ce que doit être une infirmière et, par conséquent, de la formation qu'elles doivent suivre.

\section{2-1- De la diversité des conceptions...}

Comme on l'a déjà souligné, la circulaire Combes de 1902 fait de la formation des infirmières une priorité. Ce texte est le premier qui reconnaît et définit officiellement le rôle de l'infirmière. Cette circulaire reconnaît l'infirmière comme «absolument différente de la servante employée aux gros ouvrages de cuisine, de nettoyage, etc. Elle est réservée aux soins directs des malades ». Cette division officielle permet de sortir les infirmières d'un domaine qui relèverait uniquement de la sphère domestique, et donc de revaloriser cette catégorie de personnels. De plus la conclusion de la circulaire insiste sur le caractère féminin de l'activité : «C'est une œuvre d'avenir que je vous invite à entreprendre dont les derniers termes seraient d'une part un service public convenablement outillé, d'autre part un débouché ouvert aux activités féminines sur le terrain où elles peuvent se déployer le plus utilement ${ }^{9}$. Ainsi, l'infirmière idéale est-elle définie comme une femme qui doit, aux yeux des représentants de l'État garantir un service public de qualité en matière de santé auprès de tous les malades. Pour autant, ces représentants ne disent rien de la manière concrète dont il convient de réaliser cet objectif, d'où la multiplication des conceptions en matière de formation infirmière.

Les premières formations destinées aux personnes s'occupant des soins ont été le fait de sociétés privées intervenant sur les champs de bataille. Ainsi, sont créées à la fin du XIX ${ }^{\mathrm{e}}$ siècle la Société de Secours aux Blessés Militaires (SSBM) en 1864, l'Association des Dames de France (ADF) en 1879 et l'Union des Femmes Françaises (UFF) en 1881. Cette dernière est issue d'une scission au sein de l'ADF. Ces sociétés défendent des formations courtes et théoriques, à destination des femmes issues des couches les plus aisées de la population française. Les infirmières issues de ces sociétés exercent de manière bénévole et leur 
engagement est assimilable à un devoir patriotique (16). Ces femmes sont souvent moquées pour leur « amateurisme distingué » (17).

Les premières formations à destination du personnel des hôpitaux ouvrent à l'Assistance Publique de Paris en 1878, sous la direction du docteur Désiré Magloire Bourneville. Elles prennent la forme de cours du soir municipaux, destinés au «personnel de salle» des hôpitaux parisiens. Bourneville, médecin neurologue et homme politique parisien, assure luimême une partie des enseignements. Issu d'une famille modeste, après des études de médecine, il deviendra l'assistant de Charcot à la Salpêtrière. En 1873, il fonde le «Progrès Médical », journal qui contribuera à diffuser les découvertes scientifiques novatrices et les thèses d'une médecine attentive aux questions sociales et profondément anticléricale. Le Progrès Médical fait partie des revues qui contribueront à diffuser les idées en matière de formation infirmière de cet ardent promoteur de la laïcisation des hôpitaux. La formation du personnel de salle laïc doit permettre de remplacer le personnel religieux, souvent peu disposé à suivre les prescriptions médicales, les religieuses refusant, par exemple, de réaliser les vaccins.

Le personnel de salle des hôpitaux est issu des classes sociales les moins favorisées et son niveau d'instruction est souvent faible. Les enseignements des cours municipaux sont de ce fait répartis en deux grandes catégories : des enseignements permettant au personnel de salle d'obtenir le Certificat d'Études Primaires (CEP) ; et des enseignements - théoriques et pratiques - plus spécifiques au monde du soin traitant principalement de l'hygiène, de la réalisation de pansements simples, de petite pharmacie, mais aussi de techniques plus avancées comme les sondages urinaires, les injection hypodermiques ou les techniques de vaccination, ainsi que de quelques notions sommaires d'anatomie et de physiologie et enfin de l'administration et de l'organisation hospitalière (18). La première partie des cours doit permettre aux personnels de salle d'acquérir un niveau d'étude général minimal. Dans la seconde partie, les cours spécifiques sont pensés avant tout comme des enseignements techniques où les pratiques de soins sont passées en revue avec minutie alors que les notions médicales restent survolées. Il s'agit, en même temps, de former les infirmières aux nouvelles techniques de soins et de bien circonscrire leur activité. Bourneville pense que les infirmières doivent bénéficier d'une formation pratique, mais que leur savoir théorique doit être limité. En contrôlant l'accès au savoir médical, Bourneville, tout comme nombre de ces confrères médecins, souhaite préserver le monopole de leur activité, récemment acquis ${ }^{10}$. Dans ces formations, l'accent est mis sur l'importance pour l'infirmière d'être une aide du médecin, compétente techniquement - par la maîtrise des règles d'hygiène et d'asepsie - mais aussi dévouée aux malades, comme aux médecins et à l'administration (18).

Cependant, les résultats de cette expérience ne sont pas concluants ; les personnels, éreintés par de longues journées de travail, ont des difficultés à assimiler les contenus des enseignements. Par ailleurs, l'objectif de ces cours fait l'objet de violentes critiques : le remplacement des religieuses par un personnel laïc, formé à la hâte, s'effectuerait au détriment de la qualité des soins. Le principe de mutation du personnel servant en personnel soignant, même à la condition d'une formation, est loin de faire l'unanimité dans les diverses écoles d'infirmières créées au début du XXe siècle.

Face aux critiques et surtout à leurs résultats médiocres, les cours municipaux s'ils existent encore, perdent en influence au profit de l'Ecole de la Salpêtrière. Ouverte en 1907, elle offre aux futures infirmières une véritable formation en deux ans, comprenant une initiation aux notions élémentaires d'anatomie et de physiologie, de solides connaissances techniques

10 En France, ce monopole a fait l'objet d'une lutte qui s'étend sur près d'un siècle. Elle débute par la loi du 10 mars 1803 qui instaure la notion «d'exercice illégal de la médecine » et se poursuit en 1892 avec la suppression des officiers de santé (6). 
acquises lors de stages pratiques dans divers services de l'hôpital et une éducation morale. Deux points marquent une différence entre l'Ecole de la Salpêtrière et les écoles privées : le principe du recrutement reste celui d'une ouverture démocratique; et les programmes contiennent des enseignements relatifs aux préceptes ménagers. A l'Ecole de la Salpêtrière, ces enseignements sont considérés comme essentiels pour que les infirmières puissent encadrer le personnel servant.

Parmi les détracteurs des cours municipaux, on compte la docteure Anna Hamilton (19). Issue d'une famille d'aristocrates britanniques protestante installée en France, elle sera la première femme à entrer à la faculté de médecine de Marseille. Elle convaincra un banquier de financer son travail de thèse et réalisera une enquête sur l'organisation des soins hospitaliers et sur les pratiques soignantes. Durant ce travail, elle se rapprochera de Florence Nightingale. La parution de sa thèse, en 1900 (20), viendra alimenter les débats sur la formation des personnels soignants. Elle y défend une conception des infirmières à la fois professionnelles, chargées de l'organisation des soins et disposant d'un statut égal à celui du médecin. Pour réaliser ce projet, elle intègre la Maison de Santé Protestante à Bordeaux en 1901 et y développe un modèle de formation qui s'oppose presque point par point au modèle des cours municipaux de son confrère parisien. L'idée de cette école - privée, payante et dont les enseignements sont suivis en internat - est de recruter des jeunes filles « de bonne famille », qui ont déjà une solide éducation de base et des aptitudes intellectuelles et morales. La discipline est stricte et le célibat est de mise. Les enseignements portent sur les soins et l'organisation hospitalière. Toutefois, là encore, l'accès au savoir médical est restreint. L'école est inspirée du modèle de la nurse anglaise construit et porté par Florence Nightingale. Ce modèle prône une attention particulière à l'environnement du patient : l'hygiène en fait partie, mais également le calme, l'éclairage et ce que les patients ou leurs proches peuvent exprimer. Il se différencie ainsi du modèle porté par les médecins français et permet de développer un territoire d'intervention propre aux infirmières. Hamilton milite pour la reconnaissance de celles qu'elle nomme les «garde-malades» comme les égales du médecin. Si cette appellation a aujourd'hui une connotation négative, à l'époque, elle permet de distinguer les femmes formées à Bordeaux au sein de l'ensemble hétérogène des infirmières. Provenant exclusivement de classes aisées, les élèves pourront constituer une «élite » parmi les personnels des hôpitaux, chargée de l'organisation des soins, à l'image de ce qui se fait en Angleterre. Hamilton recrute également des jeunes femmes ayant un niveau d'éducation élevé, c'est-à-dire ayant au minimum un brevet supérieur, le baccalauréat, voire une licence. De solides connaissances en langues étrangères sont également appréciées (21). D'après elle, une telle élite peut garantir la qualité des soins auprès des malades, et elle déplore que des soignantes de condition modeste soient médiocrement formées dans les cours municipaux. Elle porte également un regard critique sur les infirmières des sociétés de la Croix-Rouge, regrettant leur manque de professionnalisme.

Une autre conception des soins et de la formation des infirmières s'oppose à la formation anticléricale de Bourneville, celle de Léonie Chaptal. Elle appartient à une famille noble française, impliquée dans la vie politique de son pays. Bien que catholique elle-même, elle se revendiquera toujours comme une partisane de la laïcité. Son parcours et ses prises de position ont été facilités par ses relations dans le milieu politique et économique et par sa fortune personnelle. Au début des années 1900, elle s'investit dans des œuvres sociales du quartier de Plaisance, un faubourg parisien (21). Elle souhaite venir en aide aux familles modestes de ce quartier, en construisant des logements à loyers modérés et en améliorant la salubrité des logements existants. Elle fait également ouvrir un hôpital local, des consultations médicales et un dispensaire antituberculeux. C'est là qu'elle prend conscience de la nécessité d'un travail d'éducation à l'hygiène auprès des familles modestes, à leur domicile, dont la mise en œuvre relève d'une moralisation des classes populaires. Les mères de famille étant les premières 
destinataires de ces services, les femmes sont mieux acceptées et obtiennent de meilleurs résultats que les intervenants masculins. Chaptal s'intéresse alors de près aux infirmières dans lesquelles elle voit les actrices d'une amélioration des soins extrahospitaliers.

Après avoir elle-même suivi deux formations d'infirmières - à la SSBM et dans les cours municipaux $^{11}$ - pour se faire une idée des modèles existants, elle ouvre, en 1905, la MaisonÉcole d'infirmières privées à Paris. Elle adopte le terme d' «infirmière » pour désigner les soignantes formées dans son école qui, comme celle d'Hamilton, est socialement sélective. Son idée est «non seulement de donner une instruction professionnelle la plus complète et la plus perfectionnée possible, mais encore de relever le niveau moral de l'infirmière en recrutant [les] élèves dans des milieux de bonne éducation et de haute tenue morale » (18). La sélection est toutefois moins drastique que dans l'école d'Hamilton : les élèves - beaucoup moins nombreuses qu'à Bordeaux - sont recrutées parmi les détentrices du Certificat d'Études Primaires et ont bénéficié d'une éducation ménagère dans leur famille (22). Les enseignements, d'une durée de deux ans, sont suivis en pensionnat. Ils sont délibérément orientés vers la pratique et le manuel de référence est l'adaptation d'un ouvrage d'une infirmière britannique. Les stages pratiques, réalisés directement au lit du malade, ont une grande importance dans l'enseignement. C'est là, plutôt que dans les salles de cours, que les infirmières apprennent le métier. Cependant, contrairement au modèle développé par Hamilton, la Maison-École n'est pas directement rattachée à un hôpital. Elle est indépendante. Les stages sont réalisés dans différents hôpitaux parisiens à la fois publics et privés.

Ces différentes formations divergent sur les modalités de recrutement, sur le contenu des enseignements, et in fine sur la position que doivent occuper les infirmières dans la division du travail de soin. Cette hétérogénéité de modèles se maintient notamment du fait du peu d'investissement financier et juridique de l'État dans ces questions. Une première structuration officielle va cependant voir le jour après la Première Guerre mondiale, sous l'impulsion de Léonie Chaptal.

\section{2- 3- ... à l'unification des diplômes}

La Première Guerre mondiale contribue à la valorisation de l'image de l'infirmière, notamment par l'action des sociétés venant en aide aux blessés de guerre. De plus, après la Première Guerre mondiale, les nombreux problèmes sanitaires deviennent des enjeux démographiques cruciaux. C'est là une opportunité pour faire reconnaître la nécessité d'une régulation et d'un encadrement des formations pour les infirmières. Ces dernières ont en effet un rôle majeur à jouer dans le maintien en bonne santé de la population, notamment masculine, décimée par les années d'un conflit qui s'est éternisé.

Chaptal dispose d'un grand nombre d'atouts qui vont lui permettre d'imposer sa conception de la formation infirmière. Ces connaissances dans le domaine de la santé représentent un atout majeur. En effet, depuis le début de ses œuvres sociales dans le quartier de Plaisance, elle s'intéresse tout particulièrement au traitement des épidémies de tuberculose. Les connaissances qu'elle acquiert et les bons résultats de ses infirmières-visiteuses lui permettent d'intégrer notamment le congrès international de lutte contre la tuberculose, dont elle sera membre dès 1903 (21). Cette nomination lui confère une légitimité auprès des médecins et des pouvoirs publics. Elle tire également avantage de son insertion dans les milieux politiques et notamment de ses relations amicales avec Jeanne Millerand, la femme du Président de la République et avec Paul Strauss qui sera successivement président du Conseil supérieur de l'assistance publique (CSAP) puis ministre de l'Hygiène, de l'Assistance et de la Prévoyance sociale. Le troisième de ces atouts est la façon dont elle est perçue par les représentants des

\footnotetext{
${ }^{11}$ Elle rédigera d'ailleurs des compte-rendu de son expérience aux cours municipaux, une formation qu'elle juge de piètre qualité.
} 
pouvoirs publics. Si Chaptal est catholique, elle fait en sorte d'être perçue comme une partisane de la laïcité, notamment du fait des partenariats qu'elle développe avec l'International Council of nurses (ICN) à majorité protestante. Elle est appréciée pour sa modération et ses capacités de négociatrice : elle ne remet pas en cause la domination des médecins dans le monde de la santé.

Chaptal sera nommée au CSAP en 1913 et militera en son sein pour obtenir un titre régulant l'exercice infirmier. Cette institution joue en effet un rôle consultatif et de contrôle important dans le domaine de la santé. En 1921, Chaptal rédige un rapport expliquant la nécessité d'une implication plus forte de l'État dans la régulation de l'exercice, en garantissant une formation de qualité. Chaptal milite pour une formation rapide en deux ans pour le personnel exerçant déjà dans les hôpitaux. La conception de l'infirmière qu'elle développe n'a rien de révolutionnaire, elle emprunte des éléments aux différents modèles de formation existants. Elle est proche des écoles privées par l'importance qu'elle accorde à la bonne éducation de base des infirmières. Ces dernières doivent pouvoir justifier d'un niveau d'éducation minimal. Elle insiste également sur l'importance de l'enseignement moral au sein des écoles, ce qui lui permet de contrer les craintes des religieuses qui accusent largement les infirmières d'être des mercenaires. Enfin, elle reprend l'argument des médecins selon lequel c'est en raison des évolutions des connaissances médicales, et notamment la découverte des germes, que les techniques de soins ne peuvent plus être laissées à des amatrices. Cette formation doit être essentiellement pratique. L'accès aux savoirs médicaux menacerait en effet la profession médicale dans la mesure où les médecins exercent encore eux-mêmes de nombreuses activités de soins. Pour contrer cette critique, Chaptal affirme à plusieurs reprises que le rôle de l'infirmière est distinct de celui du médecin, et qu'elle doit lui être soumise. Elle reprend une séparation, entre maladie et malade, déjà existante dans les représentations du monde du soin. Toutefois, elle fait plus que l'énoncer, elle conforte une relation de domination en plaçant les médecins du côté de la science. Ces derniers pourraient donc s'appuyer sur un corps constitué de savoirs théoriques et techniques, et étayer leurs décisions sur des raisonnements logiques et objectifs, tandis que les infirmières, positionnées du côté de l'art, sont assignées à la sensibilité et à la subjectivité. Sans dire que cette dichotomie ait été établie en toute connaissance de cause, elle contribue à renforcer la naturalisation des compétences infirmières et à ne leur reconnaître in fine que des qualités, supposément féminines, dans ce qui constitue leur spécificité.

En 1922, plusieurs brevets de capacité professionnelle sont créés. Ils donnent le droit de porter le titre d'infirmière diplômée de l'État français. Celui-ci est accordé « aux infirmières hospitalières, aux visiteuses d'hygiène sociale et à diverses infirmières à spécialité restreinte : puériculture, surveillance sanitaire des écoles, hygiène mentale, etc. ${ }^{12}$. On reconnaît l'influence de Chaptal dans la place réservée aux visiteuses et à leurs activités de prévention et de repérage des problématiques sanitaires effectuées directement à domicile. Le décret stipule deux conditions nécessaires pour obtenir l'un des brevets de capacité professionnelle : l'infirmière doit pouvoir justifier d'une formation pratique dans une des écoles reconnues par le ministère, ainsi que de la réussite d'un examen terminal. La composition du jury d'examen terminal permet d'apprécier le poids de la profession médicale dans la formation des infirmières. Il doit en effet être composé d'au moins un médecin ou chirurgien professeur des universités, d'un ou plusieurs médecins praticiens en exercice, d'une ou plusieurs représentantes d'écoles d'infirmières et d'une infirmière en exercice.

L'article 3 du décret instaure un Conseil de perfectionnement des écoles d'infirmières (CPEI) qui aura pour mission principale d'apporter des modifications et des améliorations aux programmes et aux enseignements. Ce conseil est composé de vingt-cinq membres, nommés 
par le ministère, pour au minimum trois ans. Jusqu'en 1937, Chaptal en est la vice-présidente, tandis que la présidence est assurée par son ami, le Professeur Letulle. Le CPEI fournit également un avis consultatif sur l'autorisation des écoles à délivrer un, ou plusieurs, des brevets de capacité. Le CPEI contrôle les modalités de recrutement des élèves ainsi que celles des professeurs et des chargés de cours ; la nature et la durée des enseignements théoriques et pratiques ; le fonctionnement des internats; et les modalités d'insertion des élèves sur le marché de l'emploi. Le CPEI joue donc un rôle important dans l'harmonisation des formations permettant d'obtenir le titre d'Infirmière diplômée de l'État français. Si la décision finale revient au ministère, les avis du Conseil sont très souvent suivis et les travaux qui y sont menés conduiront à l'uniformisation nationale du programme des études dès 1924. La durée minimale de formation est fixée à deux ans, et la première année est commune aux visiteuses et aux hospitalières. Ce Conseil est une instance importante puisqu'il permet aux infirmières d'avoir un droit de regard sur les modalités de la formation, bien que ce droit doive être partagé avec les médecins.

Chaptal est parvenue à faire valoir auprès des pouvoirs publics une conception relativement unifiée de la formation, par-delà les différences entre les écoles. L'unification nationale des programmes en 1924 est à ce titre une réelle avancée. Le titre d'Infirmière diplômée de l'État français, évalué et reconnu au niveau national, constitue la première étape de la structuration $\mathrm{du}$ groupe professionnel, autour d'une autorisation d'exercer, une licence ${ }^{13}$ (23). Il n'a toutefois pas encore un caractère obligatoire. Le décret de 1922 précise que lors de l'embauche, le choix doit se porter «de préférence»sur des infirmières brevetées. Le titre d'infirmière ne ferme donc pas le marché du travail.

\section{2-3- Rendre la formation obligatoire pour protéger l'activité professionnelle Conception bénévole versus conception professionnelle}

Le travail d'unification $\mathrm{du}$ groupe professionnel ne passe pas uniquement par la reconnaissance légale d'un titre. Une autre conception de l'activité de soin est toujours très présente dans l'après-guerre, celle des tenantes d'un catholicisme intégral qui militent pour le maintien de l'influence traditionnelle de l'Eglise, y compris au niveau politique. Ces infirmières sont issues des formations des sociétés d'assistance aux blessés de guerre (SSBM, ADF, UFF). Ces associations sont composées d'aristocrates qui défendent un exercice bénévole de l'activité au nom d'un engagement d'utilité publique. Soulignons qu'elles peuvent par ailleurs compter sur les revenus de leur famille (parents ou conjoint) pour subvenir à leurs besoins. Une partie de ces infirmières, revendiquant leur appartenance au catholicisme et, récusant la formation menant vers un emploi et une activité rémunérée naissante, se coalisent en 1921. Sous la houlette de Marie d'Airoles ${ }^{14}$, elles forment l'Union catholique des services de santé (UCSS). Via cette association, profondément confessionnelle, Marie d'Airoles entend affirmer que " pour être une bonne soignante, la vocation, la foi et la capacité d'obéissance priment sur la formation » (22, p.103). L'UCSS s'oppose farouchement au caractère obligatoire du titre d'infirmière et des brevets de capacités, qui sont le reflet de l'influence de l'Etat dans le domaine du soin. Selon les principes qu'elle défend, les soins doivent être donnés bénévolement sous la direction des médecins et avec la religion catholique comme cadre de pensée. Cette association gagnera en puissance jusqu'en 1939 elle comptera alors 14000 adhérentes - avant de s'engager sur la voie du déclin dans les

\footnotetext{
${ }^{13}$ La licence, au sens d'Everett Hughes, est une autorisation légale d'exercer (23).

14 Marie de Liron d'Airoles (1884-1945) est issue d'une famille aisée profondément catholique qui s'engage dans les combats contre la laïcité. Elle militera très jeune au sein de La ligue patriotique des Françaises. C'est une association catholique dont l'objectif est d'exercer une influence politique en fournissant un soutien à des candidats opposés à la laïcité. En 1914, elle s'engage comme infirmière à la SSBM et est formée sur le terrain.
} 
années 1950. Dans les années 1920, l'idéologie de la vocation (24) développée par l'UCSS constitue la principale opposition aux conceptions développées par Chaptal. Pour faire face à cette menace, cette dernière crée en 1924 l'Association nationale des infirmières diplômées de l'état français (ANIDEF). Elle en sera la présidente. Cette association affiche une neutralité confessionnelle et ne recrute que des infirmières diplômées. Cela explique en partie son faible nombre d'adhérentes : 3000 en 1932. Rapidement, elle se met en quête de soutiens

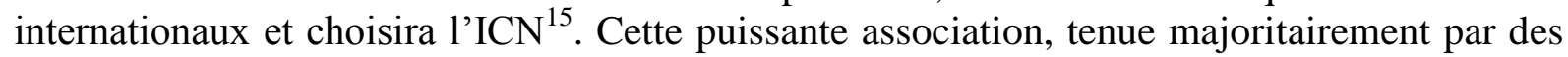
protestantes anglo-américaines, va être un soutien précieux. A partir de 1929, Chaptal devient rédactrice en cheffe de la revue nationale L'infirmière française, auparavant dirigée par des médecins. Cette revue destinée à toutes les infirmières, quel que soit leur lieu d'exercice, deviendra l'organe officiel de l'ANIDEF et un moyen pour Chaptal de diffuser sa conception du métier. De nombreux articles paraîtront pour défendre une conception professionnelle de l'activité et une amélioration des conditions de travail. Pour autant, il existe une forte opposition de la part de l'UCSS qui utilise tous les moyens officiels pour contourner la formation dans les écoles.

En effet, bien que le diplôme ne soit pas obligatoire, les pouvoirs publics prennent conscience de la nécessité de contrôler l'exercice de la profession. A cet effet, en 1925, le ministère crée le Bureau central des infirmières (BCI). Il sera notamment chargé de l'attribution des équivalences au diplôme d'État, pour les infirmières qui ne sont titulaires d'aucun des brevets de capacités. C'est notamment le cas des infirmières qui ont suivi des formations hors des écoles agréées. L'UCSS incite fortement ses adhérentes à déposer des dossiers auprès du BCI, afin d'obtenir une équivalence au diplôme délivré par l'État, alors même qu'elles n'ont pas suivi de formation dans les écoles reconnues. Les décisions, basées sur leurs états de service sont souvent favorables. L'UCSS fait pression en ce sens (25). Malgré tout, l'idée d'imposer légalement la possession du titre d'infirmière pour pouvoir exercer en France fait son chemin. En 1933, un projet de loi sur le statut des «auxiliaires médicaux », déposé à la Chambre des députés, propose la mesure. Marie d'Airoles et l'UCSS s'opposeront frontalement à ce projet, argumentant que le métier d'infirmière comporte également des tâches humbles et qu'il n'a donc pas vocation à devenir une profession qualifiée. Grâce au soutien de plusieurs députés de ses amis, le projet de loi ne sera pas présenté en Assemblée et tombera, pour un temps, dans l'oubli. Il faut également souligner que depuis la Grande Dépression de 1929, la période n'est pas économiquement propice à ce genre de mesure.

Malgré l'obtention d'un titre qui tend à unifier la catégorie émergente sous le nom « infirmière », les tensions entre les défenseurs d'un exercice bénévole et celles qui souhaitent un exercice professionnel sont encore vives. Elles freinent le processus de structuration du groupe professionnel qui peine à faire du titre d'infirmière un élément essentiel pour accéder au marché du travail. Le combat pour lier l'exercice infirmier à la détention d'un titre se poursuivra jusqu'en 1943. Cependant, pour comprendre le déroulement du processus de structuration du groupe professionnel, il nous faut nous intéresser à la scission entre le domaine sanitaire et le domaine social, véritable tournant pour le groupe professionnel infirmier.

\section{Séparation du sanitaire et du social dans l'activité infirmière ${ }^{16}$}

\footnotetext{
${ }^{15}$ La participation aux travaux de l'ICN est subordonnée à l'existence d'une association nationale. C'est parce qu'elle a créé l'ANIDEF que Léonie Chaptal peut prétendre à l'adhésion à l'ICN, toute participation individuelle étant exclue.

${ }^{16}$ Dans cette partie nous nous appuyons en partie sur les travaux d'historiennes, notamment ceux d'Eveline Dielbot (22) et d'Yvonne Knibiehler (16).
} 
Chaptal défend une conception de l'activité infirmière associant activité sociale et activité médicale. Cependant, durant les années 1930, plusieurs facteurs vont jouer contre cette conception et aboutir au renforcement de la frontière entre domaine sanitaire et domaine social.

Un premier facteur est lié aux conséquences de la Grande Dépression. Cette crise va rendre saillants des facteurs aggravants de la propagation des maladies qui n'ont rien à voir avec des causes biologiques ou médicales. Les infirmières-visiteuses, en se rendant au domicile des malades, vont prendre conscience que la propagation de maladies infectieuses comme la tuberculose sont liées aux conditions sociales d'existence des populations ${ }^{17}$. Dès lors, l'adoption de bons réflexes ne peut suffire à prévenir la propagation. Elles vont alors chercher ailleurs que dans la médecine des connaissances qui pourront leur permettre d'aider les familles à faire valoir leurs droits. Ce travail est lié à l'institutionnalisation des allocations familiales et à l'obligation des assurances sociales. Dans le même temps, l'efficacité des traitements sulfamidés rend moins primordial le travail d'éducation et de surveillance des classes populaires. Ainsi l'activité des visiteuses se détache-t-elle des considérations médicales pour se centrer sur des connaissances juridiques relatives au système de protection sociale.

Un second facteur tient aux relations entre infirmières-visiteuses et médecins. Des postes d'infirmières-visiteuses ont été créés par les médecins universitaires qui cherchaient à atteindre plus efficacement les populations qui ne se déplaçaient pas jusqu'aux dispensaires. Exerçant seules au domicile du patient, ces infirmières sont amenées à prendre des libertés dans les conseils à prodiguer, hors des directives médicales. Ces initiatives sont soutenues par les nouvelles méthodes de prise en charge et de recensement des patients enseignées dans les écoles. Les visiteuses sont toutefois rappelées à l'ordre par les médecins, et notamment par le Professeur Calmette, pourtant à l'origine du développement de leur activité. Il les met en garde contre ces velléités d'indépendance en rappelant qu'elles ne doivent sous aucun prétexte sortir de leur rôle de «collaboratrice des médecins ». Sentant une menace peser sur leur territoire d'exercice, les médecins obtiennent une limitation de l'enseignement théorique et une restriction des traitements administrés par les infirmières-visiteuses et de leur responsabilité.

Un troisième facteur tient à l'organisation des activités de soins. Henri Sellier, alors maire de Suresnes, fait le constat de la multiplication des intervenantes au domicile des patients, en partie liée aux spécialisations médicales (l'enfance, la tuberculose, les maladies vénériennes). Il propose alors un modèle polyvalent d'infirmière-visiteuse dont les interventions sont organisées par famille et non plus par type de pathologies. Cette nouvelle prise en charge a pour corollaire un changement d'autorité de tutelle. Les infirmières-visiteuses ne doivent plus seulement rendre des comptes aux médecins, mais aussi aux services de la mairie. Les médecins protestent, bien sûr, et certains refusent de s'associer au dispositif comme cela leur est proposé. Sellier fait alors appel indistinctement à des infirmières-visiteuses et à des assistantes de service social. Lorsque Sellier deviendra ministre de la Santé du Front Populaire, il tâchera de généraliser cette organisation à l'ensemble du territoire française en légiférant sur la coordination des services sanitaires et sociaux à l'échelle des départements.

Les démarches entreprises par Sellier aboutiront en 1938 à un décret ${ }^{18}$ dissociant infirmières hospitalières et infirmières-visiteuses. Ces dernières sont rattachées au corps des assistantes sociales créé en 1932. Si dans les faits, la grande majorité des assistantes sociales seront

17 Voir à ce propos, les travaux de Stéphane Henry (26) sur l'histoire et les témoignages d'infirmièresvisiteuses.

18 Décret du 18 février 1938 instituant des diplômes simple et supérieur d'infirmier ou d'infirmière hospitaliers et d'assistant ou d'assistante de service social de l'Etat. 
issues de formations d'infirmières-visiteuses jusque dans les années 1970 , le changement de nom marque de fait une séparation très nette. En préférant la dénomination «assistante sociale » à « infirmière-visiteuse », le décret replace l'activité infirmière exclusivement dans le cadre de l'hôpital, où elle se trouve totalement sous la domination des médecins. Le décret de 1938 dépossède les infirmières d'une partie importante de leur activité - le rôle social - et il restreint, provisoirement, leur exercice aux établissements hospitaliers. S'il n'occasionne pas de vives oppositions, c'est que ce décret satisfait plusieurs revendications des infirmières : 1) il instaure la sélection à l'entrée des formations ${ }^{19}$;2) il institue un diplôme supérieur pour les infirmières hospitalières, leur permettant d'exercer des fonctions d'encadrement et de formation; 3) et surtout, il stipule que le titre d'infirmière diplômée de l'État français sera exigé pour tout exercice dans un établissement public ou privé agréé, et les exercices dérogatoires sont fortement réduits.

Cependant, l'énoncé de ces règles ne suffit pas. Les établissements de santé n'ont, bien souvent, pas les moyens d'embaucher des infirmières diplômées. Malgré la réglementation, certains préfèrent mettre à profit l'expérience des personnels de salle et/ou des religieuses qui réalisent les tâches des infirmières à moindre coût. La mise en application du décret demandera encore de nombreuses années et la mobilisation de la principale association catégorielle infirmière. En 1941, l'ANIDEF constate le chômage persistant d'infirmières diplômées. Des déléguées sont envoyées au ministère de la Santé et rédigent un rapport sur la nécessité de protéger les infirmières diplômées. Elles seront entendues par le Régime de Vichy, soucieux de cantonner les femmes qui travaillent à des tâches et des travaux pour lesquelles elles seraient «naturellement» disposées. La loi du 15 juillet 1943 instaure l'obligation d'embaucher des diplômées (qu'elles soient détentrices de l'un des brevets de 1922 ou du diplôme unique de 1938). Les personnes ne possédant pas ces diplômes devront cesser toute activité dans les deux ans. L'activité des infirmières est presque exclusivement limitée au cadre hospitalier ${ }^{20}$ dans lequel la division du travail entre médecins et infirmières devient taylorienne. Les médecins s'occupent de prescrire les tâches à réaliser et les infirmières les exécutent.

A travers ce bref retour historique, il apparaît que le rôle de l'Etat est crucial, à la fois dans l'unification de la formation et dans la structuration du groupe professionnel. Les décisions de ce dernier ont été influencées par les différents protagonistes issus du monde du soin Chaptal notamment, mais aussi plusieurs médecins soucieux de la promotion de la santé publique comme Maurice Letulle, Albert Calmette, Léon Bernard, Georges Küss, Édouard Rist ou Émile Sergent - ou du monde politique - comme Sellier - ainsi que par le contexte, notamment économique, des différentes périodes. Au sortir de la seconde guerre mondiale, les infirmières sont donc très majoritairement cantonnées à un exercice hospitalier et pour la majeure partie d'entre elles, à l'autorité médicale. Toutefois, ce groupe professionnel reste très hétérogène et va connaître de nouvelles différenciations internes après la Seconde Guerre mondiale.

\section{Du modèle de la promotion sociale à celui de la promotion professionnelle ouvrant la voie à la création de postes de cheffes et à une hiérarchie infirmière à l'hôpital}

19 Les modalités d'accès aux écoles étaient déjà contrôlées par le CPEI, mais ici il s'agit d'inscrire le caractère sélectif dans la loi (Article 4, Décret ibid.)

${ }^{20}$ La loi du 15 juillet 1943 légifère également sur l'exercice à domicile pour les infirmières. Cependant ce dernier est numériquement peu important et les actes infirmiers sont là aussi soumis à la prescription médicale. 
Comme on vient de le voir, la nécessité d'une formation spécialisée, régie par un programme national, diplômante et obligatoire pour les personnels chargés du soin des malades (et non plus des indigents), notamment pris en charge à l'hôpital par des médecins, a mis du temps avant de devenir une évidence. La variété des appellations en vigueur ( garde-malade», « visiteuse d'hygiène sociale », « infirmière hospitalière », etc.) est une des manifestations de la lente et difficile institutionnalisation d'une activité relevant non plus des bonnes œuvres et du bénévolat, mais d'un métier doté d'un nom empreint, d' « une fiction mobilisatrice » (23). A sa naissance, le groupe professionnel infirmier n'est pas homogène, le personnage de l'infirmière est composite : mi-soignante, mi-assistante sociale; souvent bénévole, et quand elle est rémunérée, elle est parfois péjorativement qualifiée de «mercenaire »; laïque, mais les religieuses seront encore bien présentes au cours du $\mathrm{XX}^{\mathrm{e}}$ siècle... Une autre segmentation va encore renforcer le caractère hybride du groupe, celle de la progressive constitution d'une hiérarchisation donnant lieu à la coexistence de soignantes et de cheffes de soignantes laïques, les unes et les autres appartenant à la même famille professionnelle.

\section{3-1- Mise en place d'un dispositif de formation uniquement pensé pour les infirmières}

Si la fonction de cheffe d'infirmière «moderne » est bien pensée, voire définie par les promoteurs et pionniers au cours de la période d'émergence du groupe professionnel infirmier, la priorité immédiate n'est pas de concevoir une formation pour ces cheffes, formation qui ne pourra prendre forme et se concrétiser qu'à partir du début des années 1950 , c'est-à-dire environ 30 ans après la promulgation des brevets de capacité professionnelle d'infirmière en 1922, et presque une décennie après l'instauration de son caractère obligatoire en 1943. Toutefois, la question des cheffes n'était pas totalement inexistante au début du XX siècle.

Si dans la circulaire de 1902, Emile Combes promeut avant toute chose la création d'écoles et la formation obligatoire pour l'infirmière, il n'en oublie pas pour autant les cheffes d'infirmières qui sont évoquées et situées en deux lieux : d'une part, à la tête des écoles : «Il faudra rechercher une bonne directrice : c'est d'elle dont en très grande partie que dépendra le succès de l'école. Il importe donc de lui faire une situation convenable permettant le choix. »; d'autre part, à l'hôpital où une carrière, comprenant rémunération, avancement professionnel et retraite, est prévue : «il est désirable qu'en haut de l'échelle, et pour diriger le personnel, l'on place une femme. En bas de l'échelle, et pour les gros ouvrages, l'on emploiera des serviteurs et des servantes. $»^{21}$.

Ce monde infirmier est uniquement conçu au féminin, puisque les hommes n'ont de place que dans la catégorie des «serviteurs ». De ce fait, le genre détermine ici le sexe de la personne placée en haut de l'échelle hiérarchique, et la qualité de la directrice garante du « succès de l'école » n'est en rien due à une formation et à des diplômes réservés aux cheffes. Ses compétences ne sont pas définies, mais son appartenance à la catégorie de sexe féminin est un critère essentiel, notamment parce que l'encadrement de femmes par une femme semble aller de soi, même si in fine elles demeurent toutes placées sous l'autorité du médecin-homme (praticien dans les hôpitaux, enseignant dans les écoles).

Quant au décret de 1922, s'il institue les brevets de capacité professionnelle permettant de porter le titre d'infirmière diplômée de l'Etat français, il ne stipule aucunement la place, le rôle et la fonction de directrice d'école, de formatrice (monitrice) ou encore de surveillante de services hospitaliers.

\section{3- 2- Discours sur la formation des cheffes et projets inaboutis}

\footnotetext{
${ }^{21}$ Circulaire $n^{\circ} 7038$ du 28 octobre 1902, dite « circulaire Combes », parue au Journal Officiel de République française le 30 octobre 1902, p. 7043.
} 
Les acteurs moteurs de la constitution du groupe professionnel infirmier se sont pour certains prononcés sur la place des cheffes, mais leurs points de vue s'avèrent, tout comme au sujet des infirmières, divergents.

Bourneville prône la création d'un «monde infirmier » unifié par une filiation directe entre les infirmières et leurs cheffes, ou surveillantes, les secondes émanant du groupe des premières en suivant un parcours institutionnellement tracé, c'est-à-dire une carrière fondée sur un principe républicain de promotion sociale et professionnelle. Ses écoles jouent ainsi un rôle central dans cette progression en offrant instruction et formation à un personnel issu des couches les plus modestes de la société ainsi que des postes de gradées, inscrits dans une grille de rémunération. Bourneville ira jusqu'à prévoir une formation pour ces personnels gradés :

«Dès 1881, il a l'idée d'adjoindre à l'école de la Salpêtrière, une école à la Pitiée ${ }^{22}$, plus médicalisée, qu'il présente comme une école de perfectionnement destinée à une élite, que ce soit pour l'encadrement ou pour les soins : "Il est nécessaire que dans la mesure la plus large possible, l'Administration fasse venir ici les meilleures élèves de la Salpêtrière, celles qu'elle a l'intention de nommer suppléantes. C'est également à la Pitié, dans le personnel ainsi composé, qui serait un personnel d'élite, qu'elle pourrait recruter tous les 3 ou 4 mois, une partie du personnel destiné aux hôpitaux qu'elle va successivement laïciser." (27, p.26). En 1887, dans un article du journal Le Progrès médical', c'est la compétence technique des surveillantes laïques qu'il met en avant pour les distinguer des religieuses, occasion pour identifier quelques-unes de leurs anciennes et nouvelles activités. Les attributions les plus récentes viennent compléter et non annuler les précédentes, hormis celles qui relèvent d'un exercice d'inspiration confessionnelle, sans rapport immédiat avec le service des malades : "De même que les sœurs, les surveillantes laïques doivent surveiller la répartition des aliments, régler les rapports avec la lingerie, veiller au maintien de l'ordre et de la discipline de la salle, mais encore elles ont le devoir de demeurer constamment dans les salles, en dehors des heures des repas, tandis que les religieuses en distrayaient une large part pour la prière, la méditation, les pieux exercices de tout genre, etc. Elles doivent participer personnellement aux soins donnés aux malades, faire ou faire faire devant elles tous les pansements, toutes les petites opérations que les médecins ou les chirurgiens leur confient; prendre des renseignements précis sur les malades, noter la température, etc. En un mot, elles doivent être les premières infirmières du service, être prêtes à accomplir toutes les besognes, même les plus répugnantes." $(27, \mathrm{p} .53)$. » $(28, \mathrm{p} .122)$

A la même époque, Hamilton, autre pionnière du groupe professionnel des infirmières déjà rencontrée plus haut, défend une figure de l'infirmière «moderne » que l'on peut qualifier d'élitiste, privilégiant une main-d'œuvre issue d'une classe sociale élevée. La promotion sociale n'est pas un sujet d'intérêt pour elle, en revanche elle envisage comme une évidence l'accès à des fonctions de cheffes pour ses nurses formées. Transférant le modèle anglais de Nightingale en France, elle met en place des postes de «sous-cheftaines » et de «cheftaines » et confie à ce personnel gradé l'encadrement d'élèves en formation et de garde-malades en activité.

A l'école, « une cheftaine encadrait les élèves pendant les cours théoriques, lesquels étaient assurés par les médecins et professeurs de médecine qui fréquentaient la Maison de Santé protestante ou qui étaient agréés par elle. Les cheftaines assuraient 1'“intendance", c'est-àdire : contacts, accords, fonctionnement, planification, progression des enseignements selon les années de présence, programmes, etc. Elles corrigeaient les copies des élèves, tandis que

22 Il s'agit alors du premier hôpital de la Pitié, dans le $5^{\mathrm{e}}$ arrondissement de Paris, qui sera détruit et remplacé par l'actuel hôpital de la Pitié, construit au début du $\mathrm{XX}^{\mathrm{e}}$ siècle à proximité de la Salpêtrière. 
les professeurs les interrogeaient au début des cours supplémentaires ou désiraient en savoir plus sur un sujet ou un autre. » $(29$, p.58)

A l'hôpital, ce sont non seulement des fonctions de cheftaines que les nurses doivent occuper, mais aussi celle de directrice d'établissement telle que la définit Hamilton : " $L a$ 'Directrice', voilà un personnage encore à peu près inconnu en France, et que la réforme hospitalière introduit graduellement dans les établissements réorganisés. Cette individualité est une conséquence forcée de la création d'un personnel infirmier de moralité et d'éducation supérieures constitué principalement par des jeunes filles qui ne peuvent être placées que sous une direction féminine"23. Le Dr Anna Hamilton, quant à elle cumule les fonctions de directrice de l'école de garde-malades et de directrice de l'œuvre de la Maison de santé protestante. » $(29, \mathrm{p} .61)$

Ces directrices trouveront à s'embaucher dans des hôpitaux publics ou privés, où elles exerceront souvent, en plus des tâches de soins, des fonctions d'encadrement. Afin de défendre sa conception de la formation des «garde-malades », Hamilton crée en 1908 le Conseil national français des directrices d'hôpitaux (CNFDH). Cette association a pour but de défendre le travail hautement qualifié des garde-malades qui assurent des fonctions de directrices des hôpitaux. Cette association s'inscrit dans les mouvements féministes des premières femmes avocates et médecins. Nombreuses sont les adhérentes qui considèrent que la seule façon d'exercer dans le domaine de la santé, sans occuper une place subalterne, c'est de constituer une profession autonome et exclusivement féminine (22, p.269). Cette conception des soins ne rencontre que très peu d'écho en France et les adhérentes de l'association seront dispersées suite à la Première Guerre mondiale.

Tout comme les promoteurs de l'émergence du groupe professionnel infirmier, Hamilton réserve ce métier aux femmes, mais l'importance qu'elle donne à l'appartenance à une classe sociale favorisée ne la conduit pas à cantonner les infirmières dans un rôle maternel d'exécutrice, mais plutôt à leur ouvrir des perspectives professionnelles moins dominées en convoquant les compétences sexuées attendues dans leur univers bourgeois: ainsi, elle «pense qu'il est primordial d'avoir un personnel uniquement féminin, afin que les élèves vivent dans un milieu conforme à la moralité, et parce qu'en outre seules les femmes sont capables par leur éducation d'accomplir certaines tâches, comme celle de diriger une maison : "L'hôpital est dirigé par une ancienne garde-malade d'expérience reconnue, laquelle s'occupe, comme le ferait une 'bonne maîtresse de maison', de tout ce qui se passe dans son intérieur" ${ }^{24} \gg(29, p .60-61)$ Les croyances d'Hamilton sur la place des femmes sont tout aussi sexistes que celles de Bourneville, mais fondées sur d'autres justifications en concordance avec son appartenance de classe supérieure où des femmes (bourgeoises ou "patronnes») savent gérer leur intérieur et commander d'autres femmes placées sous leurs ordres (30).

\section{3- 3- Des discours aux effets limités sur l'avènement des cheffes infirmières}

Ces discours sont novateurs parce qu'ils contribuent à construire les fondements d'un groupe professionnel pour des femmes et, ce faisant, à leur octroyer une place dans la division sociale du travail et sur un marché du travail où elles peuvent occuper un emploi rémunéré. Toutefois, le segment des cheffes restera encore longtemps dans l'ombre du groupe professionnel infirmier où il ne parviendra à éclore que tardivement.

Le modèle de Bourneville, promouvant la laïcisation, la qualification et la promotion d'un personnel féminin soignant hospitalier, se généralisera en France, mais bien après la fin du $\mathrm{XIX}^{\mathrm{e}}$ siècle : «En 1890, la totalité des hôpitaux de 1'Assistance publique de Paris étaient laïcisés, exceptés l'Hôtel Dieu, Saint-Louis et Berk-sur-Mer. D'après les chiffres établis par J. 
F. Piera (31), huit hôpitaux étaient déjà laïcs avant la période que nous avons envisagée [1870-1900], dont Bicêtre et la Salpêtrière. Six nouveaux établissements furent confiés à des laïques dès leur origine et dix-huit furent laïcisés entre 1878 et 1888. Cependant, la laïcisation était restée un phénomène marginal et parisien. Si quelques hôpitaux comme ceux de Roubaix, Marseille, Meulan et la Rochelle suivirent l'exemple, 90\% des hôpitaux français demeuraient religieux. » (27, p.54)

Quant au modèle d'Hamilton, il ne fera pas l'unanimité, et sera jugé élitiste, trop exigeant et sans doute quelque peu effrayant, voire subversif, du fait de la place qu'elle entend donner aux femmes à l'hôpital, aux côtés des hommes médecins, allant jusqu'à leur octroyer des responsabilités hiérarchiques ou des fonctions de direction, tout comme les matrons qui occupent la fonction la plus élevée dans la hiérarchie hospitalière infirmière des hôpitaux anglais.

En 1938, la question de la formation des cheffes refait son apparition dans de nouveaux textes, mais toujours en arrière-plan des évolutions du groupe professionnel des infirmières qui connaît alors un tournant, celui de la distinction entre infirmières hospitalières et assistantes sociales. Ce même décret prévoit également la délivrance de «diplômes supérieurs » par des écoles supérieures de service hospitalier et sociale. Mais l'heure de la reconnaissance institutionnelle n'a pas encore sonné pour les cheffes d'infirmières : "Il est possible de déceler dans ce projet, les prémices d'une formation de "cadres" et notamment d'enseignantes pour les écoles d'infirmières. L'initiative ne voit pas le jour immédiatement en raison de la guerre. En 1942 toutefois un décret en date du 10 août institue le diplôme d'Etat de monitrice (32). Ce texte sera validé après la Libération sans être lui non plus suivi d'effets. » (28, p.126).

La création officielle d'une formation pour cheffes-infirmières ne verra le jour officiellement qu'au lendemain de l'après-guerre à travers l'ouverture d' " écoles de cadres »: "A la veille de la Seconde Guerre mondiale, où 40000 infirmières de la Croix-Rouge française vont s'engager, l'orientation de l'enseignement est axée vers la population en péril. Il faudra attendre 1951 pour voir la création de la première école de cadres des soins infirmiers par la Croix-Rouge française. » (1, p.28). L'offre de formation comprend un double programme d'études : l'un destiné aux infirmières qui se destinent à l'enseignement dans les écoles d'infirmières, l'autre aux infirmières briguant un poste de surveillante à l'hôpital, concrétisant ainsi une forme de spécialisation au sein du segment des cheffes.

Dans les années cinquante, d'autres ouvertures d'écoles ont lieu en France, à l'Assistance publique de Paris, à Toulouse ou encore à Strasbourg (33). Toutes ces formations sont sanctionnées par un diplôme d'école. Elles s'adressent à des infirmières expérimentées et confirmées qui se perfectionnent dans leur domaine. Il s'agit de la diffusion du modèle de Bourneville fondé sur la promotion professionnelle et l'ancrage de la fonction de cheffe dans le métier infirmier d'origine.

Le décret $\mathrm{n}^{\circ}$ 58-1104 du 14 novembre 1958 parachève ce processus par la création de deux certificats d'aptitude aux fonctions d'infirmière surveillante et d'infirmière monitrice (CAFIS et CAFIM), délivrés à l'issue d'une formation de huit mois. De ce fait, la hiérarchisation du groupe infirmier s'établit sur un dédoublement de la catégorie de cheffes, ajoutant, ou renforçant, une segmentation supplémentaire interne entre les «cadres » appelées surveillantes en service hospitalier et les «cadres» appelées monitrices ou directrices en école. Une nouvelle sous-division est encore instaurée par le décret de $1973^{25}$ qui porte création du certificat d'infirmière cadre de santé publique : les trois grands espaces de travail des infirmières sont désormais investis par une fabrication réglementée de leurs cadres : les écoles, l'hôpital et l'extra-hospitalier. 


\section{3- 4- Amorce d'une hiérarchisation du groupe professionnel infirmier fondée sur les classifications en corps et grades de la fonction publique}

A l'origine conçu, défini et façonné par des non-infirmiers (médecins, hommes politiques, philanthropes, législateurs, etc.) ayant mobilisé leurs valeurs de classe, morales et religieuses, le groupe professionnel infirmier a progressivement été peuplé par ses propres travailleuses, porteuses d'une identité professionnelle naissante qu'elles ont cherché à développer, transformer, défendre avec et contre d'autres professionnels de champs voisins. Une élite infirmière va se constituer et, grâce à ses ressources notamment culturelles et sociales, s'engager dans un travail militant. Parallèlement, un besoin social de cadres se fait sentir. L'ouverture et l'augmentation du nombre des écoles d'infirmières, puis de cadres; le développement d'associations professionnelles nationales et internationales en plein essor dès les années 1920 ; la création de postes institutionnels dans différents conseils et instances ministérielles (Conseil de perfectionnement, Bureau central des infirmières, etc.) offrent des places de représentantes à occuper (34). Enfin, le développement de l'activité hospitalière (35), notamment à partir des années 1950, entraîne non seulement des besoins de maind'œuvre infirmière, dont la pénurie est récurrente pendant des décennies, mais aussi d'encadrantes de ce personnel.

Dans l'après-guerre, les infirmières bénéficient donc d'un vent porteur, notamment parce que leurs compétences sont recherchées tant au niveau national qu'international dans un contexte de reconstruction puis d'essor économique où la santé publique est une question sociale et politique centrale. Une occasion favorable à leur groupe professionnel va se présenter à elles, et pendant trente ans des avancées notables pourront se produire.

\section{3-5- Un épisode marquant dans le cours de l'histoire du groupe professionnel infirmier ${ }^{26}$}

C'est au début des années 1960 qu'un acteur clé entre en scène : dans le cadre de la décolonisation, l'Organisation mondiale de la santé (OMS) définit une politique d'aide des pays riches européens aux pays de la région africaine dits en état de sous-développement. Les soins curatifs et préventifs faisant cruellement défaut, le personnel infirmier se trouve situé au centre des programmes sanitaires. Ces orientations vont favoriser un développement de la profession d'infirmière et encourager une élévation du niveau de leurs compétences. C'est dans cette optique que l'OMS vise la constitution d'une élite professionnelle composée d'infirmières hautement qualifiées, destinées à occuper des postes à responsabilité, soit dans l'enseignement, en tant que directrices d'école, soit dans les services hospitaliers, en tant que surveillantes générales, chargées de l'organisation des soins infirmiers.

Un processus d'enrôlement (37) va alors se mettre en place et recouvrir la constitution d'un réseau d'acteurs et l'instauration de négociations multilatérales afin de parvenir à l'aboutissement du projet d'une école internationale d'enseignement supérieur pour infirmières (EIEIS) : à l'OMS s'ajoutent la Fondation Rockefeller (surtout active dans l'entredeux guerres), l'Etat français via le ministère de la Santé publique et la Population, les Hospices civils de Lyon (HCL), et l'université de Lyon, avec quelques-unes de ses facultés. L'implication de ces cofinanceurs et décideurs va permettre l'ouverture de l'EIEIS. Au passage, l'Ecole nationale de la santé publique (ENSP), lieu de formation des directeurs d'hôpital, est écartée du projet, signe que les temps ne sont pas encore à la «managérialisation» des futures cheffes d'infirmières. Preuve en est le contenu du programme d'enseignement sur deux années qui comprend une très grande part de sciences

26 Ce paragraphe s'appuie sur le travail de recherche mené par Michel Poisson ; le lecteur pourra se référer à sa thèse afin d'y trouver une analyse contextualisée et approfondie de l'histoire de l'EIEIS (36). Le document est consultable en ligne : https://tel.archives-ouvertes.fr/tel-01951900/document 
humaines et sociales (philosophie, psychologie, anthropologie, sciences politiques, pédagogie, etc.), les apports médicaux étant par ailleurs intégrés dans les enseignements infirmiers (comme «en soutien » aux soins infirmiers, démarche inverse à celle adoptée à l'origine et conservée pendant longtemps dans les programmes de formation pour infirmières). Quant à l'intitulé du diplôme, il affiche à lui seul la revendication d'une identité professionnelle : « DU d'enseignement infirmier supérieur ».

Promoteurs, financeurs et personnels nourrissent de grands espoirs à l'ouverture de l'Ecole et profitent de vents porteurs. En toile de fond, des politiques publiques en faveur de la formation professionnelle promotionnelle notamment conduites par Michel Debré ; une période dite des Trente glorieuses, dont la gloire toute relative (38) est tout de même propice aux engagements de dépenses hospitalières, facilitant en l'occurrence l'engagement des HCL qui alimentent grandement le budget de l'Ecole ; un soutien intéressé du ministère de la Santé préoccupé par la pénurie d'infirmières dans les établissements et qui manque de formatrices (appelées monitrices) dans les écoles qu'il crée pour pallier cette pénurie ; une université qui commence à ouvrir plus grand ses portes à de nouveaux profils d'étudiants et, enfin, un groupe professionnel en attente de revalorisation de ses études, de la constitution des sciences en soins infirmiers en tant que discipline et d'un accès à la recherche grâce à la création d'un doctorat. Tout concorde donc pour que l'expérience soit couronnée de succès, et les débuts sont prometteurs.

Au cours des premières années, grâce aux moyens dont ils disposent, les membres de l'Ecole vont développer une intense activité intellectuelle de militantes, d'enseignantes et de quasichercheures universitaires. De nombreux déplacements en France ou à l'étranger leur sont notamment financés par l'OMS, tels que des voyages d'étude, des missions d'experts en soins infirmiers ou encore des «séminaires résidentiels ». Elles mènent une activité associative en tant qu'élues dans des organismes collectifs et instances permanentes (commissions, conseils de perfectionnement, comités d'entente) des écoles d'infirmières et de cadres infirmières, produisent des rapports et participent à des enquêtes sur le métier d'infirmière, sont membres de commissions ministérielles. Certaines d'entre elles vont même accéder à des postes à responsabilité au ministère. Par ailleurs, les anciennes élèves de l'Ecole vont mettre sur pied un réseau, et créer, en 1972, l'AMIEC (association des amis de l'EIEIS), puis, en 1975, les Cahiers de l'AMIEC, consacrés à l'étude des soins et du service infirmier. Par le biais de ces initiatives, un essaimage de «l'esprit EIEIS » va se produire, et cet effet sera prolongé par les diplômées de l'Ecole qui deviendront monitrices ou directrices d'écoles de cadres ou d'infirmières. «L'esprit EIEIS» constitue le soubassement d'un éthos professionnel en construction de «l'infirmière moderne", qui imprègne sa pratique de principes moraux défendant entre autres : une prise en charge globale du patient, c'est-à-dire, parallèlement (ou contrairement) aux médecins, du malade et non seulement de la maladie; l'éducation thérapeutique du patient, acteur de son parcours de soins; le développement d'une politique de la santé publique tournée vers le hors-hospitalier, etc.

Toutefois, une difficulté majeure se pose d'emblée, elle restera sans solution et sera fatale à l'Ecole : le nombre des inscriptions d'étudiantes, notamment françaises, est trop faible. Est-ce le signe d'un manque d'attractivité ? Oui, mais la qualité des enseignements ne peut être mise en cause. L'OMS ne finance des bourses que pour les étudiantes étrangères (et encore, ces financements vont progressivement diminuer), et les Françaises ne sont pas soutenues par leur employeur, parce que les débouchés professionnels n'ont pas été mis en place. Dès avant l'ouverture de l'Ecole, les promoteurs avaient défendu la généralisation et l'institutionnalisation à l'hôpital de postes de surveillantes générales, et la promulgation de textes imposant une formation après concours, dispensée... par l'EIEIS.

Tout espoir est perdu en 1975, date de création du corps des infirmières générales. Dans les conditions d'accès, la réglementation n'impose pas l'obligation de suivre une formation 
supérieure à l'Ecole, mais un temps d'adaptation à l'emploi assuré par... l'ENSP ! Les négociations avec le ministère et la ministre de l'époque, Madame Simone Veil, n'ont pas connu d'issue favorable pour les tenants de l'EIEIS. De surcroît, la même année, l'Assistance publique de Paris ouvre les portes de son $\mathrm{IESCH}^{27}$, institut de formation supérieure destinée à ses cadres hospitalières, délivrant une maîtrise de sciences et techniques, en partenariat avec l'université de Paris-Dauphine. Et c'est ainsi, que l'EIEIS perd en 1975 son monopole sur le marché de la formation supérieure des infirmières, et ses possibles débouchés sur le marché professionnel des cadres infirmières.

Dès 1978, la survie de l'Ecole est en question. Après un changement de direction administrative, confrontée au tournant gestionnaire qui s'annonce à partir de 1970, année d'une loi hospitalière instaurant une logique de restrictions financières, les HCL vont devenir très exigeants et en attente de rentabilité : l'EIEIS et ses personnels sont sommés de dégager des ressources propres et pour ce faire de développer leur activité de formation professionnelle continue. L'EIEIS disparaît et devient un département d'enseignement infirmier supérieur (DEIS) d'une part installé au sein d'un IIFSCS ${ }^{28}$ des HCL, et d'autre part rattaché à l'université de Lyon. Le montage institutionnel, qui peut paraître compliqué de l'extérieur, fait perdre de sa visibilité à l'ex-Ecole et au passage son caractère international. La liberté d'action des personnels du DEIS est réduite. Ceux-ci sont contraints d'assurer une certaine productivité en tant que fournisseurs de sessions de formations continues de courte durée, plus rentables que leur service dans le DU d'enseignement infirmier supérieur, rescapé de la restructuration, mais toujours aussi peu attractif.

Tout au long de ses dix-sept années d'existence (de 1978 à 1995), le DEIS va poursuivre ses activités de formation, les membres tentant de rester fidèles au projet fondateur. Les rapports avec la direction de l'hôpital seront tendus jusqu'au bout. Le soutien de l'OMS faiblit pendant que le ministère délaisse le développement des soins infirmiers, au profit de l'enseignement de la gestion et du management pour tous ses cadres, paramédicaux y compris. La démotivation s'installe au sein des personnels de l'ex-Ecole, ils ne parviendront pas à adhérer aux nouveaux objectifs organisationnels, et faute de rentabilité, le DEIS s'éteint en 1995.

La logique professionnelle a, en l'occurrence, été vaincue par la logique organisationnelle et gestionnaire. L'Ecole d'enseignement supérieure, dont les membres ont lutté pour l'accès à l'université des infirmières et la création d'une discipline en sciences infirmières, n'a pas survécu aux impératifs financiers doublés d'un manque de soutien ministériel. Le «top management » (directrices des soins, ex-infirmières générales) de la hiérarchie infirmière et paramédicale est de ce fait aujourd'hui formé à l'EHESP ${ }^{29}$, ex-ENSP, là même où sont formés les directeurs d'hôpitaux. La fermeture de cette Ecole, restera un événement marquant dans le cours de l'histoire des infirmières en France, mais sans pour autant en interrompre le cours qui va s'éloigner de l'idéologie de l'EIEIS pour se mettre au goût du jour, et s'orienter vers des perspectives plus libérales, managériales et gestionnaires.

\section{4- Une élite « managérialisée », « gestionnarisée »}

La piste des évolutions des dispositifs de formation des infirmières et plus précisément de leurs cheffes, comme révélatrice des dynamiques de leur groupe professionnel, nous amène à un nouveau tournant, celui d'une conversion managériale et gestionnaire en adéquation avec les transformations de l'hôpital. Les cheffes d'infirmières sont à plusieurs reprises rebaptisées, parallèlement aux changements introduits dans leur fabrication et socialisation. L'« universitarisation » tant souhaitée par les équipes de formatrices de l'EIEIS se produit:

27 IESCH : Institut d'enseignement supérieur de cadres hospitaliers.

28 IISFCS : Institut international supérieur de la formation des cadres de santé.

29 EHESP : Ecole des hautes études en santé publique. 
comme on l'a vu plus haut, les infirmières obtiennent un grade de licence ; puis c'est au tour des cadres de santé d'atteindre le niveau master (1 ou 2). Enfin, malgré une "réingénierie » du diplôme de cadres de santé «en cours » (stagnante au niveau du ministère depuis 2011), les choses avancent sur le terrain. Alors qu'avec un temps de retard des auteurs de rapports prônent une managérisation des cadres, les établissements publics se sont déjà inspirés des méthodes du privé : comme nous le verrons plus loin, DRH et directrices des soins mettent en place des démarches de repérage d'agents «à potentiel», de «talents » dont ils gèrent la carrière par le biais de dispositifs tels que les postes de faisant fonction, s'immisçant ainsi dans les interstices de la réglementation hospitalière.

\section{4- 1- Des surveillantes/monitrices aux cadres de santé : 1975, 1995}

Depuis 1958, les formations de cheffes délivrant deux certificats, CAFIS et CAFIM, établissaient formellement une distinction entre deux activités, conduisant certains agents à dire que monitrices et surveillantes ne faisaient pas le même métier, position encore publiquement défendue aujourd'hui par des représentants d'associations professionnelles alors que ces deux catégories de cadres ont été fusionnées en 1975 par un décret ${ }^{30}$ qui promulgue le «certificat de cadre infirmier» (CCI). L'amorce d'une polyvalence professionnelle se poursuit dans le milieu des années 1990: la logique de filiation professionnelle entre les infirmières et leurs cheffes, notamment instaurée dès la fin du XIX siècle par Bourneville, est remise en question : ce lien est distendu par un décret ${ }^{31}$ qui transforme et élargit les conditions d'entrée dans la profession d'encadrant. Le diplôme est désormais délivré à des « cadres de santé » (le titre de «cadre infirmier » tombe en désuétude) et cette formation est ouverte à l'ensemble des professionnels paramédicaux réglementés. De ce fait, la formation devient potentiellement accessible à un plus grand nombre de professionnels non seulement issus de la filière infirmière, mais aussi des filières de rééducation et médico-technique ${ }^{32}$.

Enfin, à partir de 2001, ce diplôme devient obligatoire pour exercer la profession de cadre de santé, tout au moins dans le secteur public. De ce fait, pour les cadres de santé, la promotion à l'ancienneté disparaît ${ }^{33}$. Il n'existe plus qu'un seul mode d'accès au grade, celui du concours sur titre, interne ou externe, ouvert aux candidats titulaires du diplôme de cadre de santé ou d'un certificat équivalent. Autrefois classés dans la catégorie B de la fonction publique hospitalière, les cadres de santé accèdent à la catégorie $\mathrm{A}$ et bénéficient d'une grille indiciaire revalorisée.

L'évolution du contenu de la formation reflète également la métamorphose qui s'opère sur la catégorie des encadrants : le nouveau programme de 1995 «parachève une orientation totale de la fonction vers une dimension gestionnaire amorcée dans les programmes précédents. Une comparaison des quatre programmes officiels montre la diminution de la place de la clinique dans cette formation. A partir de 1995, les savoirs se rapportant à la médecine ne sont plus enseignés et sont remplacés par des savoirs liés à l'économie de la santé et à la gestion. Les connaissances en médecine et en chirurgie ne sont plus testées lors du concours d'entrée en

30 Décret $n^{\circ} 75-928$ du 9 octobre 1975.

31 Décret n95-926 du 18 août 1995 portant création d'un diplôme de cadre de santé, JO 20 août 1995, n¹2468.

32 Trois filières sont concernées, chacune comprenant plusieurs spécialités : 1) filière rééducation : diététicien, ergothérapeute, masseur-kinésithérapeute, orthophoniste, orthoptiste, pédicure-podologue, psychomotricien ; 2) filière soins : infirmier, infirmier de secteur psychiatrique, infirmiers spécialisés ; 3) filière médico-technique : audioprothésiste, manipulateur d‘électroradiologie médicale, opticien-lunetier, préparateur en pharmacie hospitalière, technicien de laboratoire d'analyses de biologie médicale.

33 Décret $\mathrm{n}^{\circ}$ 2001-1375 du 31/12/2001, titre Ier article 2, portant statut particulier du corps des cadres de santé de la fonction publique hospitalière 
Institut de formation des cadres de santé (IFCS), alors qu'elles constituaient l'essentiel du programme de l'année préparatoire. » (39, p.37).

Les glissements sémantiques qui ont remanié le titre des encadrants constituent symboliquement une autre manifestation de cette conversion des professionnels consacrés à la surveillance en managers des temps modernes hospitaliers. L'introduction du terme « cadre » s'est faite en conservant dans un premier temps la référence au métier (« cadre infirmier », en 1975), avant de l'abandonner, comme pour marquer l'intégration dans la catégorie plus large des cadres («cadres de santé » à partir de 1995) et le rapprochement avec les cadres administratifs et techniques intervenant dans les établissements de soin.

Les cadres de santé se voient aujourd'hui attribuer des missions qui relèvent des domaines de la gestion et du management. Si l'on se réfère à la fiche de poste figurant dans le Répertoire des métiers de la fonction publique ${ }^{34}$, quatre grandes dimensions d'activité peuvent être dégagées : la gestion de ressources humaines (organisation des services, coordination, suivi des personnels), la gestion des moyens matériels et financiers (contrôle, reporting, tableaux de bord, etc.), la conduite de projets (rapports d'activités, veille, etc.), la garantie de la qualité des soins (sécurité, hygiène, protocoles, etc.). On notera que le travail de soin est totalement absent de cette liste de tâches prescrites et que le terme de patients n'apparaît pas une seule fois.

\section{4- 2- Une formation cadre professionnelle doublée d'une formation universitaire}

Cette conversion au management et à la gestion n'a pas été souhaitée et impulsée que par les employeurs qui compteraient sur de cadres de santé performants afin de satisfaire leurs attentes d'efficience, d'amélioration de la qualité des soins et de contrôle des dépenses selon les principes dit du new public management (NPM) (40), dans un contexte économique de plus en plus contraint.

L'élite intellectuelle des infirmières, dont faisaient partie les membres de l'EIEIS, revendique depuis longtemps un rapprochement avec l'université et l'obtention d'un diplôme universitaire et non pas seulement professionnel. Comme on l'a vu, l'EIEIS avait établi un partenariat avec l'université de Lyon et l'AP-HP avec l'université de Dauphine, via l'IESCH, dès les années 1970. Sans pouvoir dater précisément le début du processus, les écoles de cadres (devenues IFCS) ont pour leur part noué des liens avec différentes facultés. Le texte réglementant le diplôme de cadres de santé de 1995 a d'ailleurs officiellement encouragé ce mouvement que les formatrices et directrices avaient déjà initié, les textes entérinant après coup des initiatives émanant du terrain.

\section{Arrêté du 18 août 1995 relatif au diplôme de cadre de santé}

Art. 23. - Les organismes gestionnaires des instituts de formation des cadres de santé sont habilités à établir, par convention, un partenariat avec les universités pour:

1- L'enseignement de modules du diplôme de cadre de santé, de la licence des sciences de l'éducation et de la licence des sciences sanitaires et sociales;

2- La participation d'enseignants relevant du ministère chargé de l'enseignement supérieur aux évaluations de ces modules ;

3- La prise en compte de modules du diplôme de cadre de santé dans le cadre de la licence des sciences de l'éducation et de la licence des sciences sanitaires et sociales, et de modules de la licence des sciences de l'éducation et de la licence des sciences sanitaires et sociales dans le cadre du diplôme de cadre de santé.

Une nette progression est notable depuis les textes 1995. L'article 23 de l'arrêté de 1995 sur les conditions de mise en œuvre de la nouvelle formation de cadre de santé ne cite que deux

34 Consultable à cette adresse : http://www.metiers-fonctionpubliquehospitaliere.sante.gouv.fr/pdf/metier.php? idmet $=27$ 
disciplines susceptibles d'intéresser les IFCS. Un peu plus de vingt ans après, l'intérêt pour le partenariat entre écoles professionnelles et université persiste, et s'est encore davantage institutionnalisé et étendu au point de faire l'objet d'un rapport en 2017 intitulé : "Pour une meilleure intégration des formations paramédicales à l'université : mise en œuvre des mesures 5, 6, 13 de la Grande conférence de santé » (41). Placé en annexe, un « recensement des partenariats universitaires - IFCS et leur niveau M1/M2 », réalisé par le Comité d'entente des formations infirmières et cadres (CEFIEC) fin 2016, nous apprend que la formation de cadres de santé s'est non seulement « universitarisée », mais également «mastérisée » : sur 36 IFCS répertoriés en France, 35 délivrent au moins un diplôme universitaire parallèlement au diplôme de cadres de santé. Le niveau M1 est encore majoritaire, mais la relative concurrence existant sur le marché de la formation des cadres de santé poussent les IFCS à offrir un M2 en faisant évoluer leur convention avec les universités et en aménageant leur contenu de formation, c'est-à-dire en renforçant le couplage des programmes du diplôme professionnel et des masters. La majorité des masters sont spécialisés dans le management (54\%), alors que loin derrière, les sciences de l'éducation ne représentent que $22 \%$ de l'offre.

Tableau 1 : Partenariat IFCS et universités

\begin{tabular}{|c|c|c|c|c|}
\hline & \multicolumn{3}{|c|}{ Parallèlement au diplôme professionnel de cadre de santé : } \\
\hline $\begin{array}{c}\text { Nombre } \\
\text { d'IFCS }\end{array}$ & $\begin{array}{c}\text { Diplômes universitaires } \\
\text { délivrés au niveau M 1 }\end{array}$ & $\begin{array}{c}\text { Diplômes universitaires } \\
\text { délivrés au niveau M 2 } \\
\text { N=36 }\end{array}$ & $\begin{array}{c}\text { Autre diplôme } \\
\text { universitaire délivré }\end{array}$ & $\begin{array}{c}\text { Aucun diplôme } \\
\text { universitaire }\end{array}$ \\
\hline Par 22 IFCS & $\begin{array}{c}\text { Par 12 IFCS } \\
\text { (dont un en cours de } \\
\text { constitution) }\end{array}$ & Par 1 IFCS & Par 1 IFCS \\
\hline
\end{tabular}

D'après les formatrices d'écoles de cadres interviewées, les sciences de l'éducation étaient très prisées jusque dans les années 1990, puis elles ont perdu du terrain au profit des sciences de la gestion et du management. On peut faire l'hypothèse que cette évolution suit celle du milieu infirmier et hospitalier : nombre de formatrices et de directrices d'écoles de cadres ont, dans un premier temps, donné le ton, puis progressivement, notamment avec la diffusion du NPM, une partie des infirmières et surtout leurs cheffes ont été imprégnées par l'idéologie managériale, attirées par ou poussées vers les opportunités professionnelles offertes par les hôpitaux sous forme de postes de manager ("cadre de santé, "cadre supérieure », « infirmière générale » rebaptisée «directrice des soins », puis «cadre paramédicale de pôle », «cadre transversale », etc.). Un article pourrait à lui seul être consacré aux manifestations de cet engouement managérial observable chez les cheffes d'infirmières. Il faudrait notamment s'intéresser aux discours dépréciatifs tenus à propos des fonctions de formatrices $^{35}$, vues comme des «fausses cadres » face aux «vraies cadres » en service, ou encore aux modèles de «bons cadres » ou plus exactement de «bons managers » que des revues professionnelles véhiculent à travers la mise en valeur des innovations organisationnelles et projets managériaux mis en place par des cadres à l'hôpital. Ces investigations devraient également interroger le malaise des formatrices en IFCS, prises entre les finalités de leur mission: former des bons cadres correspondant aux attentes des employeurs, relayées par les cheffes des personnels paramédicaux, et leur vision plus distanciée, voire critique, du rôle dévolu aujourd'hui aux cadres paramédicales, ex-cheffes d'infirmières à l'hôpital.

35 Nos enquêtes de terrain révèlent que la place des formatrices, en IFSI comme en IFCS, n'est pas aussi légitime que celle des cadres en service, et que nombre de professionnels tels que DRH, directrices des soins, cadres de santé mettent en doute leurs compétences et utilité. Un rapport officiel, publié en 2010, a soulevé un tollé en tentant de réformer les formations de cadres hospitaliers et en mettant en cause les compétences de formatrices trop éloignées du terrain pour former correctement les cadres (42). 


\section{4- 3- Des cadres de santé aux cadres-manager : une réingénierie en suspens depuis le début des années 2010}

De manière générale, ces expériences de terrain, rejoignent une tendance nationale de transformation du statut réglementaire des cadres dont l'amorce visible et rendue publique peut être située au moment de la « Mission cadres hospitaliers » confiée en 2009 par Roselyne Bachelot, alors ministre de la santé, à Chantal de Singly ${ }^{36}$, ex-directrice d'hôpitaux parisiens, et alors directrice de l'Institut de management à l'Ecole des hautes études de la santé publique. Le rapport qu'elle a remis en septembre 2009 dresse un état des lieux de la situation des cadres de santé à l'hôpital public, mais émet également de nombreuses propositions regroupées en six rubriques :

«1- Pour une réelle politique managériale au sein de chaque établissement,

2- Pour une reconnaissance universitaire de la formation des cadres et le renforcement du lien avec le métier ${ }^{37}$,

3- Pour une dynamique régionale du développement des compétences managériales et de soutien des cadres,

4- Pour un accompagnement national des projets et des innovations par les cadres,

5- Pour valoriser les niveaux de responsabilités des cadres par les statuts et les rémunérations, 6- Pour porter et piloter les suites de la mission ».

Les intitulés parlent d'eux-mêmes et traduisent l'orientation donnée à ce rapport. Son auteure $\mathrm{y}$ insiste fortement sur la dimension managériale de la fonction de cadre de santé, qu'elle entend revaloriser, notamment pour répondre aux griefs des cadres de terrain consultés, qui se plaignaient d'un manque de reconnaissance et de marges de manœuvre, et s'estimaient insuffisamment rémunérés.

Ces inflexions s'inscrivent dans le cours d'une série de réformes hospitalières, et notamment de celle de 2005 qui a instauré la «nouvelle gouvernance des établissements publics de santé ${ }^{38}$. A cette occasion, les pôles d'activité et la tarification à l'activité (T2A) ont été mis en place. A la tête de l'organisation dite "polaire » de l'hôpital est placé le "trio de pôle » composé d'un chef de pôle, d'un cadre supérieur de santé et d'un cadre de gestion. Le "pilotage médico-économique " de l'hôpital s'appuie sur un "principe de subsidiarité » censé favoriser « la transmission de l'autorité et des marges de décision aux échelons les plus proches de la production de soins. » $(43$, p. 3$)$.

Officiellement, aucune évolution ne s'est produite pour les cadres de santé, le texte de 1995 reste en vigueur. Mais de nombreux signes, nettement perceptibles sur le terrain, contredisent le constat d'Isabelle Feroni et Anémone Kober-Smith qui affirmaient en 2005 : «En France, la permanence du rôle de l'Etat en matière d'emploi, au travers du statut de la fonction publique hospitalière, garantit l'homogénéité des règles de recrutement, des appellations d'emploi et des salaires, à la différence de la Grande-Bretagne où les réformes des années quatre-vingt ont permis aux établissements de diversifier les profils de postes dans le contexte d'une nouvelle grille salariale de la profession. » (44, p.484). D'une part, les formations de cadres de santé connaissent des évolutions vers l'universitarisation et la mastérisation non encadrées réglementairement, générées par des influences diverses mais convergentes: attentes des acteurs de terrain (direction des écoles, professionnelles, étudiantes infirmières, etc.), objectifs des établissements, évolutions universitaires sous l'effet LMD (licence, master,

36 Chantal de Singly, après avoir travaillé dans les collectives locales, a été directrice de l'hôpital Laennec, de l'hôpital Trousseau et de l'hôpital Saint Antoine à Paris. Depuis avril 2010, elle est directrice générale de l'Agence régional de santé de l'Océan Indien.

37 Sous-entendu : métier de cadre et non d'infirmière.

38 Ordonnance $\mathrm{n}^{\circ}$ 2005-406 du 2 mai 2005 simplifiant le régime juridique des établissements de santé. 
doctorat) venant de l'Europe, politique nationale de santé, etc. D'autre part, la gestion des carrières des agents hospitaliers se transforme et les employeurs d'établissements publics s'inspirent des méthodes du privé, par exemple en tentant d'exercer un contrôle sur la promotion professionnelle, se faisant notamment par voie de concours, de leurs futures cadres en imposant des parcours formalisés à leurs agents entre autres contraints de passer par des «statuts » de faisant fonction de cadres et de suivre des formations-maison et d'accepter différentes modalités d'accompagnement de parcours visant une préparation à la fonction cadre, avant ou après, en tout cas en dehors de la formation en IFCS, hors hôpital (14).

\section{5- Conclusion}

L'étude du milieu infirmier vient conforter des constats déjà établis en sociologie, selon lesquels la formation, initiale ou continue (45), est une des composantes déterminantes de la structuration d'un groupe professionnel, composante qui se décline notamment sous la forme d'écoles habilitées, de formatrices reconnues, d'un programme national d'enseignement, de conditions d'entrée plus ou moins sélectives, d'un diplôme d'Etat, d'une réglementation officielle et d'instances de contrôle du respect de la réglementation.

L'approche sociohistorique (46) permet de se prémunir contre une erreur ancienne et tenace, celle qui consiste à considérer cette «structuration» dans une perspective linéaire et évolutive, conduisant au stade final et supérieur de «profession». Et cette tentation est d'autant plus grande quand on s'intéresse aux infirmières que les catégorisations institutionnelles ont classées dans la famille des «paramédicaux » ou encore des «auxiliaires médicaux » (cf. code de la Santé, livre III). Une logique de « désir de professionnalisation » s'applique en l'occurrence facilement, dans le sens où ces infirmières n'auraient de cesse de vouloir imiter les médecins, et in fine être leurs égales. Or, d'une part, la «profession» médicale a d'abord dû se battre pour conquérir son prestige, et ce prestige s'avère fragile et de façon récurrente remis en question par des transformations, dont certaines sont perçues de l'intérieur comme dévalorisantes, par exemple à travers la féminisation du corps médical (47) ; et, d'autre part, les infirmières ont quant à elles affiché (et affichent encore) des revendications d'autonomie et de démarcation par rapport au monde des médecins. Comme eux, elles ont pu constater que rien n'est jamais vraiment acquis. Par exemple en décembre 1987, lorsque la ministre de la Santé de l'époque publie un arrêté ouvrant aux non bacheliers l'accès aux écoles d'infirmières, initiative qui, il est vrai, a mis le feu aux poudres et créé une mobilisation des infirmières sans précédent (48). Sur une plus longue période, on a vu à quel point il a été difficile de parvenir à protéger le titre d'infirmière, par le biais de l'obtention obligatoire du diplôme d'Etat, condition sans cesse remise en question par les multiples dérogations prévues dans les textes de loi. Mais aux régressions ou lenteurs, s'adjoignent des évènements interprétés comme des bonds en avant par les infirmières et leurs représentantes : l'actualité en témoigne avec la mise en place en 2018 du master d'infirmière de pratique avancée, suivie en septembre 2019 de l'annonce de la création d'une quatrième mention dite « urgences ${ }^{39}$, ajout qui vient étendre le périmètre d'intervention de ces (super)infirmières. Reste à s'interroger sur les conséquences de telles avancées : postes de médecins manquants comblés par des postes moins coûteux d'infirmières; terrain gagné par les infirmières sur celui des médecins, à la condition d'un renforcement de leur subordination à la prescription médicale ou d'un développement des principes et pratiques relevant des soins infirmiers...

39 Arrêté du 18 juillet 2018 relatif au régime des études en vue du diplôme d'Etat infirmier en pratique avancée, prévoyait trois mentions : 1) pathologies chroniques stabilisées, 2) oncologie et hémato-oncologie, 3) maladie rénale chronique, dialyse, transplantation rénale. 
Indéniablement donc l'instauration et le développement des dispositifs de formation, de préférence couronnés par des diplômes d'Etat, procurent de nombreux gains à un groupe professionnel : avantages symboliques (prestige, légitimité), économiques (négociation à la hausse des salaires), politiques (meilleure position dans les rapports de force avec d'autres groupes professionnels et avec l'Etat), etc. Mais la qualification des travailleurs, objectivée par la délivrance de diplômes obligatoires, pour exercer le métier, et inscrits dans une nomenclature organisée en niveaux a, en l'occurrence, ouvert la voie à une stratification des postes et fonctions, et à l'institution de cheffes d'infirmières, devenues avec le temps des cheffes paramédicales, pas obligatoirement infirmières à l'origine, et chargées d'encadrer du personnel soignant (majoritairement infirmier).

Ces processus de hiérarchisation ont joué un rôle important dans les évolutions du groupe professionnel infirmier (puis paramédical), composé de différentes strates de cheffes qui se sont plus ou moins coalisées, notamment lors des luttes pour la création du grade d'infirmière générale (1975), puis du service de soins infirmiers (1991), pour défendre tout à la fois leurs propres intérêts et, au moins dans leurs discours, ceux de leur famille professionnelle.

Le milieu infirmier offre un terrain propice au développement de recherches spécifiquement centrées sur le rôle des cheffes dans les évolutions d'un groupe professionnel. Nombre de questionnements jaillissent immédiatement : comment, par qui et pourquoi une hiérarchie de fonctions, fondée sur une carrière, se construit-elle? Quelles en sont les justifications (principes républicains, favorables à la promotion sociale, comme pour Bourneville, ou encore principes bourgeois misant sur la moralité d'une élite, comme pour Hamilton) ? A quoi cette hiérarchisation/stratification sert-elle, d'une part, à l'employeur, qui l'instrumentalise pour instaurer un ordre organisationnel, et, d'autre part, aux catégories de cheffes susceptibles de s'allier ou de se diviser, à l'instar des cadres formatrices et des cadres en service ? Quels sont les facteurs susceptibles de renforcer ou de relâcher les liens entre les travailleurs de différents niveaux de la hiérarchie, même lorsque la promotion n'est ouverte qu'aux membres du métier, en l'occurrence du soin ? Par exemple, dans quelle mesure les infirmières se reconnaissent-elles dans leur supérieures placées au plus haut de la hiérarchie ? Globalement, c'est toute la question du sens du concept de « groupe professionnel » qui est posée au regard de ses multiples segmentations verticales, sans oublier les divisions horizontales, dont les IPA sont peut-être porteuses?

\section{Conflit d'intérêt}

Les autrices déclarent n'avoir aucun lien d'intérêt.

\section{Références}

(1) Duboys Fresney C., Perrin G. Le métier d'infirmière en France. Paris: Presses universitaires de France; 1996.

(2) Wilensky H.L. The professionalization of everyone ? Am J Sociol. 1964 Sep; LXX(2): $137-58$

(3) Dubar C. Sociologie des professions. Paris: Armand Colin;1998.

(4) Coquillard I. L'émergence d'un groupe professionnel : les docteurs régents de la faculté de médecine de Paris au XVIIIe siècle. Dans: Histoires de nobles et de bourgeois : Individus, groupes, réseaux en France. XVIe-XVIIIe siècles [En ligne]. Nanterre : Presses universitaires de Paris Nanterre; 2011 [cité le 11 juillet 2019]. Disponible: http://books.openedition.org/pupo/3899>. ISBN : 9782821851122. DOI : 10.4000/books.pupo.3899. 
(5) Demazière D., Gadea C. Sociologie des groupes professionnels: acquis récents et nouveaux défis. Paris: Editions La Découverte; 2009.

(6) Gadéa C., Grelon A. Est-ce ainsi que les professions meurent ? Dans: Demazière D, Gadéa C. Sociologie des groupes professionnels: acquis récents et nouveaux défis. Paris: Editions La Découverte; 2009. p.118-28.

(7) Schweyer F.X. Genèse et dynamiques de l'encadrement hospitalier. Soins cadres. 2010 Aug; 19(75): 16-9.

(8) Abbott A. Écologies liées : à propos du système des professions. Dans: Menger PM. Les professions et leurs sociologies: modèles théoriques, catégorisations, évolutions. Paris: Maison des Sciences de 1'Homme; 2003. p.29-50.

(9) Bezes P. Réinventer l'Etat: les Réformes de l'administration française (1962-2008). Paris: Presses Universitaires de France; 2009.

(10) Girard L. Des femmes en blanc : la «fabrication » des infirmières. [Thèse de sociologie]. Dijon : Université de Bourgogne Franche-Comté; 2018.

(11) Girard L. «Universitarisation » des Instituts de Formation en Soins Infirmiers : enjeux et positionnement des cadres de santé formatrices. Journées d'études : pratiques coopératives et participatives en santé. Nanterre; mars 2019.

(12) Divay S. Soignantes dans un hôpital local: des gens de métier confrontés à la rationalisation et à la précarisation. Rennes: Presses de l'Ecole des Hautes Etudes en Santé Publique; 2013.

(13) Divay S. Cadres en devenir: évolutions, transformations, socialisations, tensions. Toulouse: Octarès; 2017.

(14) Divay S. La nouvelle fabrique de la carrière des cadres de santé à l'hôpital : entre réglementation et cooptation. Sci Soc Sante. 2018 Mar;36(1):39-64.

(15) Divay S, Gadea C. Aggiornamento managérial des établissements publics et dynamiques professionnelles: éléments d'analyse à partir de l'évolution des cadres de santé, Gouv. action publique, 2015;4(4):81-99.

(16) Knibielher Y. Cornettes et blouses blanches. Paris: Hachette; 1984.

(17) Senotier D. Cent ans d'évolution de la profession. Dans: Kergoat D et al. Les infirmières et leur coordination 1988-1989. Paris: Lamarre; 1992.

(18) Leroux-Hugon V. L'infirmière au début du XXe siècle : nouveau métier et tâches traditionnelles ». Mouvement Soc. 1987 Jul-Sep;(140):55-68.

(19) Dielbot E. Anna Hamilton (1864-1935), l'excellence des soins infirmiers. Rech Soins Infirm. 2017 Dec;(131): 85-100.

(20) Hamilton A.E. Considérations sur les infirmières des hôpitaux. [Thèse de médecine] Montpellier. Faculté de Montpellier; 1900.

(21) Dielbot E. Léonie Chaptal (1873-1937), architecte de la profession infirmière. Rech Soins Infirm. 2012 Jun;(109):93-107. 
(22) Dielbot E. Les femmes dans l'action sanitaire, sociale et culturelle, 1901-2001. Les associations face aux institutions. Paris: Femmes et associations; 2001.

(23) Hughes E.C. Le regard sociologique. Essais choisis. Textes rassemblés et présentés par Jean-Michel Chapoulie. Paris: Éditions de l'École des Hautes Etudes en Sciences Sociales; 1996.

(24) Suaud C. L'imposition de la vocation sacerdotale. Actes Rech Sci Soc. 1975 May;1(3):217.

(25) Dielbot E. L'Union catholique des services de santé (UCSS) et les Auxiliaires sociales (UAS) (1923-1939). Dans: Tartakowsky D, Tetart F. Syndicats et associations : concurrence ou complémentarité ? Rennes: Presses Universitaires de Rennes; 2006. p.47-54.

(26) Henry S. Histoire et témoignages d'infirmières visiteuses (1905-1938). Rech Soins Infirm. 2012 Jun;(109):44-56.

(27) Poisson M. Origines républicaines d'un modèle infirmier (1870-1900). Vincennes: Editions Hospitalières; 1998.

(28) Poisson M. Des surveillantes aux cadres de santé (1875-2015) : points de repères et éléments de réflexion sur les origines et l'évolution d'une fonction dans le système de santé français. Dans: Divay S. Cadres en devenir: évolutions, transformations, socialisations, tensions. Toulouse: Editions Octarès; 2017. p.117-47.

(29) Dielbot E. La maison de santé protestante de Bordeaux : 1863-1934: vers une conception novatrice des soins à l'hôpital. Toulouse: Érès; 1990.

(30) Rollins J. Entre femmes. Actes Rech Sci Soc. 1990 Sep;84(2):63-77.

(31) Piera J.F. Histoire de la laïcisation des hôpitaux de l'Assistance publique de Paris vue à travers le Progrès médical de 1873 à 1893. [Thèse de médecine] Créteil : Université de Paris Val de Marne; 1979.

(32) Magnon R. Léonie Chaptal, 1873-1937: la cause des infirmières. Paris: Editions Lamarre; 1991.

(33) Magnon R. Le service infirmier ces trente dernières années. Paris: Editions du Centurion; 1982.

(34) Charles G. L'infirmière en France d'hier à aujourd'hui. Paris: Le Centurion; 1979.

(35) Maillard C. Histoire de l'hôpital de 1940 à nos jours. Paris: Dunod; 1986

(36) Poisson M. L'Ecole internationale d'enseignement infirmier supérieur Lyon (19651995). Fabrique d'une élite et creuset pour l'émancipation des infirmières françaises du XXè siècle. [Thèse d'histoire]. Le Havre : Université Le Havre-Normandie; 2018.

(37) Callon M. Éléments pour une sociologie de la traduction. La domestication des coquilles Saint-Jacques dans la Baie de Saint-Brieuc. L'Annee Sociologique. 1986;36:169-208

(38) Pawin R. Retour sur les «trente glorieuses » et la périodisation du second XXe siècle. Rev Hist Mod Contemp. 2013 Jan-Mar;LX(1):155-75. 
(39) Bourret P. Les cadres de santé à l'hôpital: un travail de lien invisible. Paris: Editions Seli Arslan; 2006.

(40) Belorgey N. L'hôpital sous pression: enquête sur le «nouveau management public ». Paris: Editions La Découverte; 2010.

(41) Debeaupuis J., Allal P. Pour une meilleure intégration des formations paramédicales à l'université : mise en œuvre des mesures 5, 6, 13 de la Grande conférence de santé. Rapport IGAD n²016-123R / IGAENR n²017-043. [En ligne]. Paris; 2017 juin. [cité le 18 octobre 2019]. Disponible: https://www.ladocumentationfrancaise.fr/var/storage/rapportspublics/174000676.pdf

(42) Yahiel M., Mounier C. Quelles formations pour les cadres hospitaliers ? Rapport de l'IGAS. [En ligne]. Paris: novembre 2010.[cité le 18 octobre 2019]. Disponible: https://www.ladocumentationfrancaise.fr/rapports-publics/114000037/index.shtml

(43) Zeggar H. et al. Bilan de l'organisation en pôles d'activité et des délégations de gestion mises en place dans les établissements de santé. [En ligne]. IGAS, La Documentation Francaise; 2010. [cité le 18 octobre 2019]. Disponible: http://www. ladocumentationfrancaise. fr/rapports-publics/104000246/index.html.

(44) Feroni I., Kober-Smith A. La professionnalisation des cadres infirmiers : l'effet de l'action publique en France et en Grande-Bretagne. Rev Fr Sociol. 2005;46(3):469-94

(45) Dubar C., Gadea C., Perez C. Sociologie de la formation post-scolaire. Dans: Carré P., Caspar P., directeurs. Traité des sciences et des techniques de la formation. Paris: Dunod; 2011. p.125-42.

(46) Guibert J., Jumel G. La socio-histoire. Paris: Armand Colin; 2002.

(47) Lapeyre N., Le Feuvre N. Féminisation du corps médical et dynamiques professionnelles dans le champ de la santé. Rev Fr Aff Soc. 2005 Mar;1:59-81

(48) Kergoat D., Imbert F., Le Doaré H., Senotier D. Les Infirmières et leur coordination 1988-1989. Paris: Editions Lamarre; 1992. 